EUROPEAN ORGANIZATION FOR NUCLEAR RESEARCH

CERN PH-EP-2004-040

12 May 2004

\title{
FROM BUBBLE CHAMBERS TO ELECTRONIC SYSTEMS: 25 YEARS OF EVOLUTION IN PARTICLE DETECTORS AT CERN (1979-2004)
}

\author{
Fabio Sauli
}

CERN, CH-1211 Geneva, Switzerland

\begin{abstract}
The European Organization for Nuclear Research (CERN) celebrates in October 2004 its $50^{\text {th }}$ anniversary. A centre of excellence for elementary particle physics research, the laboratory has contributed to the development of advanced technologies for the construction of powerful accelerators and experiments. As a follow-up of the article published by this journal in 1980, on the occasion of CERN's $25^{\text {th }}$ anniversary, this contribution describes the developments of particle detectors and experimental setups that took place in the laboratory during the last twenty-five years.
\end{abstract}

Submitted to Physics Reports, CERN 50 th anniversary special issue Corresponding author: Fabio.Sauli@cern.ch (F. Sauli) 


\section{INTRODUCTION}

The European Organization for Nuclear Research $\left(\mathrm{CERN}^{1}\right)$, founded in 1954, celebrates this year 50 years of successful operation and great discoveries in fundamental particle physics. CERN's first accelerator, the $600 \mathrm{MeV}$ SynchroCyclotron (SC) started operating in 1956; after providing for years beams to physics experiments, it was dedicated to ISOLDE, CERN's isotope separator, until the shut down in $1990^{2}$. Its successor, the $28 \mathrm{GeV}$ Proton Synchrotron (PS) provided the first beams in 1959. Used for decades to supply particles to physics experiments, with various improvements it has been exploited as injector to all new machines: the Intersecting Storage Rings (ISR, 1971-83), the $400 \mathrm{GeV}$ Super Proton Accelerator (SPS, 1976 to present), the $p-\bar{p}$ collider (1981-1990), the Large Electron Positron collider (LEP, 1989-2000). From 2007, the venerable PS will inject beams into the newest CERN machine, the Large Hadron Collider (LHC), expected to begin operation almost exactly fifty years after the first; its available centre-of-mass energy, $14 \mathrm{TeV}$, will be close to six orders of magnitude larger than the one achieved by the SC.

The progress in detector technology has closely followed the commissioning of the powerful accelerator complexes. In this article, I will outline the progress of detector components and systems over the last twenty-five years, in a continuing effort to cope with new and challenging physics requirements. This note begins where ends the article written by Leon Van Hove and Maurice Jacob, celebrating the first twenty-five years of CERN and published by this journal in 1980 [1].

The special volumes "History of CERN" describe the early years of the laboratory [2].

\section{THE DECLINE OF BUBBLE, SPARK AND STREAMER CHAMBERS}

Made sensitive to ionising tracks by the rapid expansion of a super-heated liquid volume, bubble chambers visualize with trails of bubbles the trajectories of ionising particles. The Big European Bubble Chamber (BEBC) took the first pictures in 1972. Gargamelle, its heavy liquid sibling, built in Saclay and installed at CERN in 1970, was instrumental for the discovery of neutral weak currents. After many years of successful use, a failure due to metal fatigue put a stop to operation in 1978.

It became clear in the late seventies that, despite their powerful imaging capability, bubble chambers were inadequate to achieve identification of energetic particles. Additional electronic devices came temporarily to the rescue, as the External Muon Identifier [3], making use of the recently introduced Multiwire Proportional Chambers (Fig. 1). A method of holographic track recording, introduced in 1984, resulted in a fivefold improvement of resolution, and extended the lifetime of the complex. Eventually, even these improvements were not sufficient to compete with newly developed devices, and CERN's last bubble chamber was shut down in 1985.

Bubble chambers, active during pre-determined times, visualize ionising tracks without selection. Already in the sixties a competing device, the spark chamber, permitted selective recording of events, albeit with an inferior quality. Initially relying on photographic recording, spark chambers entered the electronics era with the development of ingenious automatic systems to record positions of the particle-induced discharges. Using wire planes as electrodes, the spark currents along the wires could be detected to provide positional information. A very popular

\footnotetext{
${ }^{1}$ The acronym derives from the early denomination "Centre Européen pour la Recherche Nucléaire".

${ }^{2}$ The ISOLDE program itself continues using $1 \mathrm{GeV}$ beams provided by the PS booster.
} 
method, the magneto-strictive readout, relied on detecting a sonic wave induced by sparks on a thin metallic ribbon, magnetically coupled to the wires in the sparking gaps. The author actually started his lifelong involvement with detectors winding the miniature coils used to reconvert, at both ends of the magneto-strictive ribbon, the sonic waves into electrical pulses. Alternative readout methods used ferrite cores or capacitors for information storage. Such digital spark chambers, in conjunction with other detectors providing a "look at me" flag (the trigger) represented a valuable tool for the study of rare events, and were in wide use throughout the seventies [4]. Superseded by the far more performing proportional chambers, wire spark chambers disappeared from the floor in the early eighties.

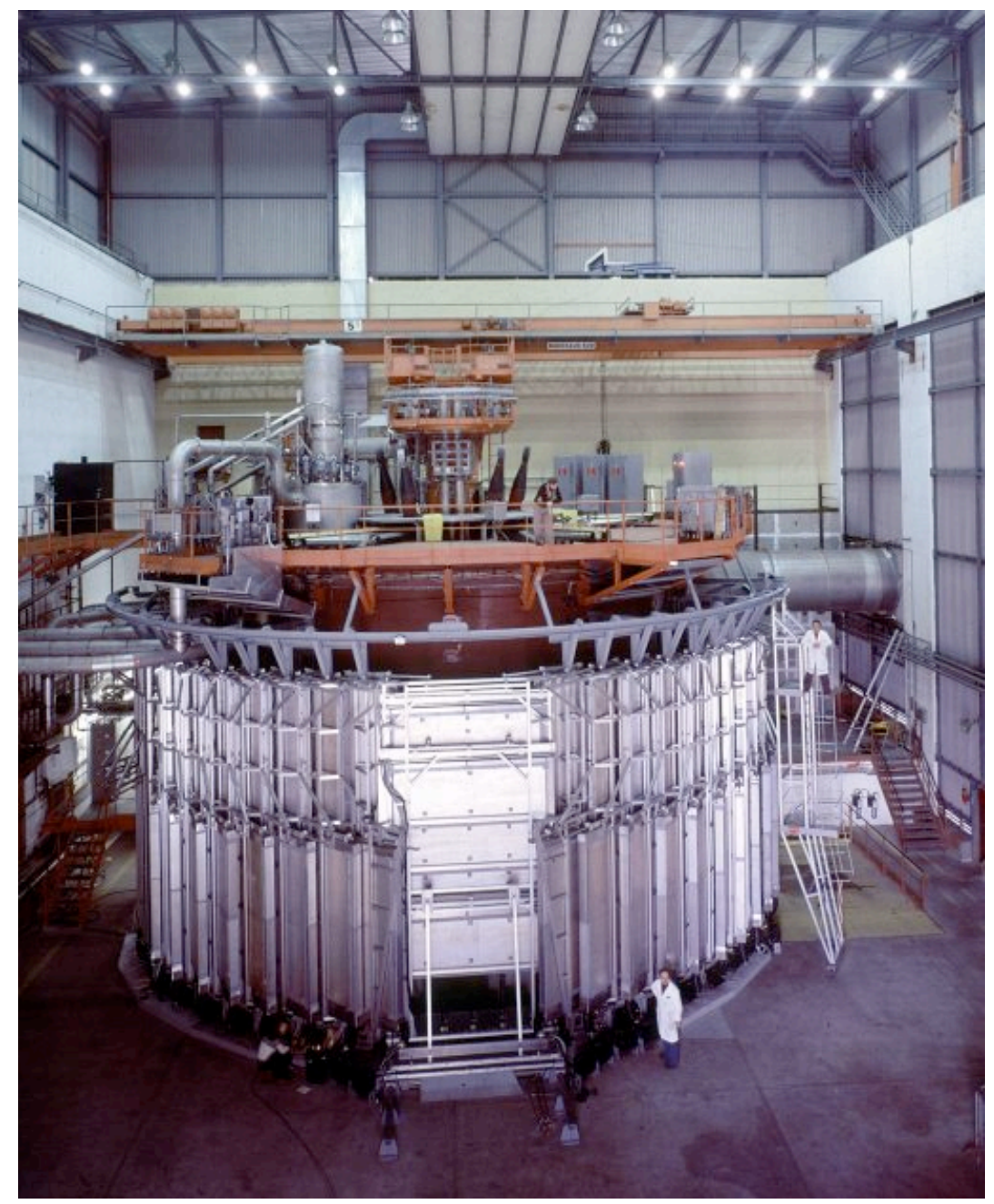

Fig. 1: The Big European Bubble Chamber with the External Muon Identifier, using gaseous electronic detectors.

Combining selective trigger functionality with excellent imaging capability, large volume streamer chambers were a competing alternative to bubble chambers, particularly for the study of events containing a large multiplicity of tracks. A short high voltage pulse applied in synchronism with the interactions forms luminous filaments along the ionisation trails; the streamers, initially witnessed photographically, were later recorded using automatic digitising cameras Fig. 2 shows the interaction of a high-energy sulphur ion hitting a gold target, recorded with the NA35 streamer chamber. At the time, no other device could have provided such a richness of information [5]. 


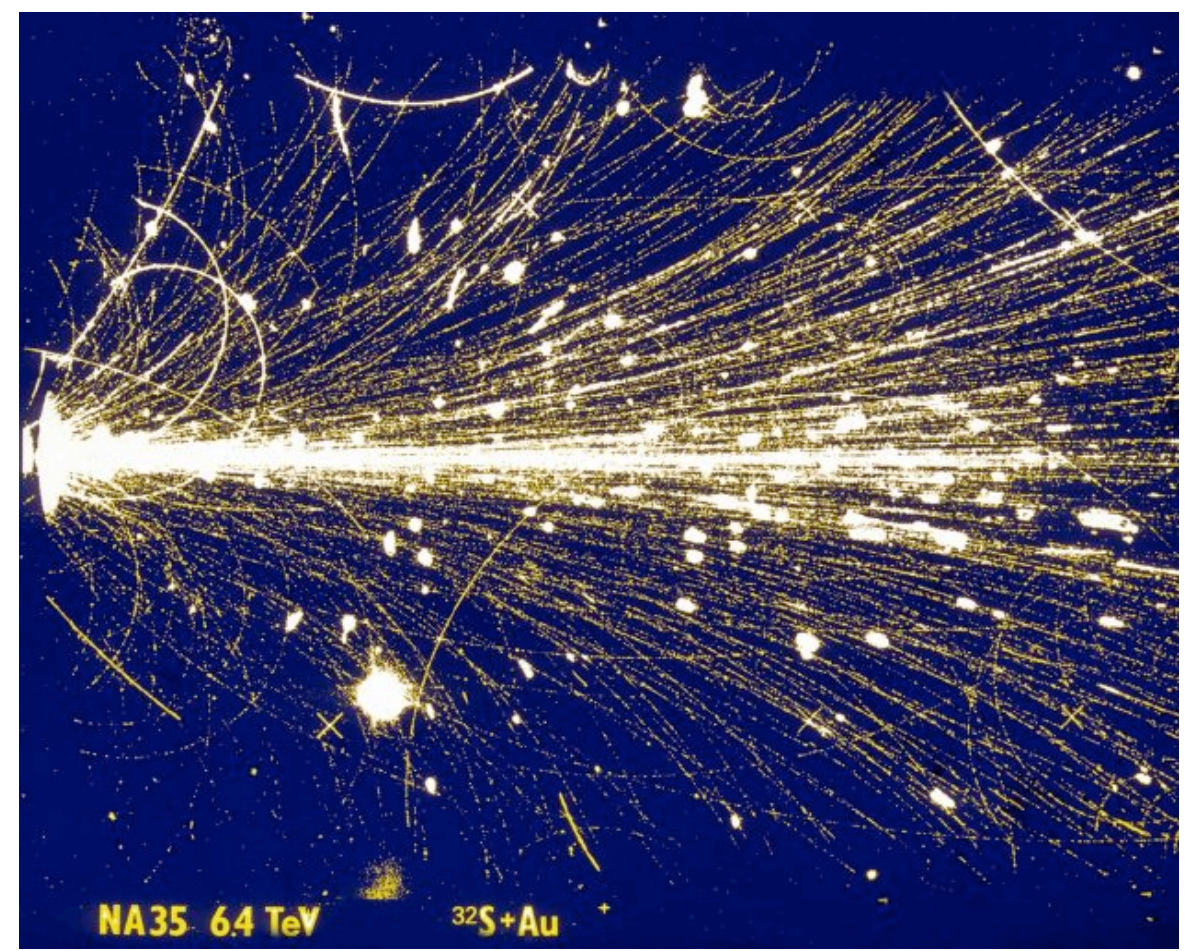

Fig. 2: Heavy ions interaction detected by the NA35 streamer chamber.

\section{THE PROPORTIONAL WIRE CHAMBER REVOLUTION}

Despite various improvements and the development of digital readout methods, spark chambers could not operate at rates above a few tens of Hertz, a rather grim limitation for the emerging physics of rare events. A true innovation in detector concepts came in 1968, with the development by Georges Charpak of the Multiwire Proportional Chamber (MWPC) [6]. A set of parallel, tin anode wires stretched between two cathode foils at high voltage, act as individual proportional counters and amplify the ionization trails released in the gas by charged particles (Fig. 3). Coupled to appropriate electronic circuitry, the chambers are continuously sensitive, able to withstand high fluxes and to localize tracks with millimetre precisions. Further studies revealed that recording the charge induced on cathodes could largely improve the detector performances, providing two-dimensional localization of tracks with accuracies of a tenth of $\mathrm{mm}$ or better [7].

The first large-size MWPCs were successfully used in the early seventies by Jack Steinberger and collaborators in an experiment on CP violation [8]. In their classic conception, the detectors are built up with a stack of insulating frames, supporting wire planes and thin-foil gas windows. Signals are extracted from the anode wires, and after the development of the induced charge readout method also from groups of cathode wires. Fig. 4 shows a large MWPC prototype, built in 1972 in Charpak's group; in the picture, the inventor stands at left and the author, recently associated to his research team, in the centre.

With a modified design, making use of light honeycomb supporting plates, a set of twenty large size MWPC constituted the core of the Split Field Magnet Detector installed at one of the intersects of CERN's proton-proton intersecting storage rings (ISR)(Fig. 5) [9]. Tracks emerging from the beam-crossing region are deflected by the magnetic field and recorded in a succession of wire planes, the curvature providing the momentum. The detector started operation in 1972 and took data, at an unprecedented rate of several $\mathrm{kHz}$, until the ISR decommissioning in 1983. 


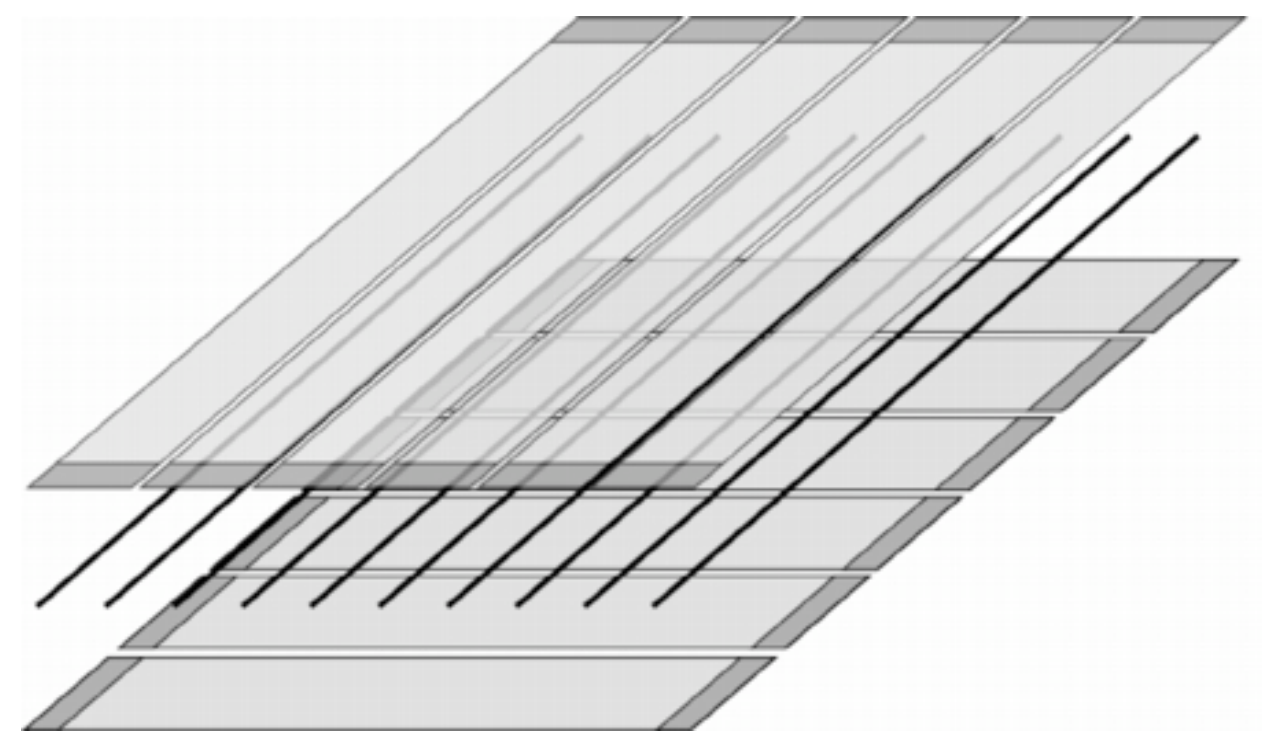

Fig. 3: Schematics of the Multiwire Proportional Chamber. Thin anode wires, between two cathodes, act as independent proportional counters. Recording the induced signals on segmented cathodes offers two-dimensional localization.

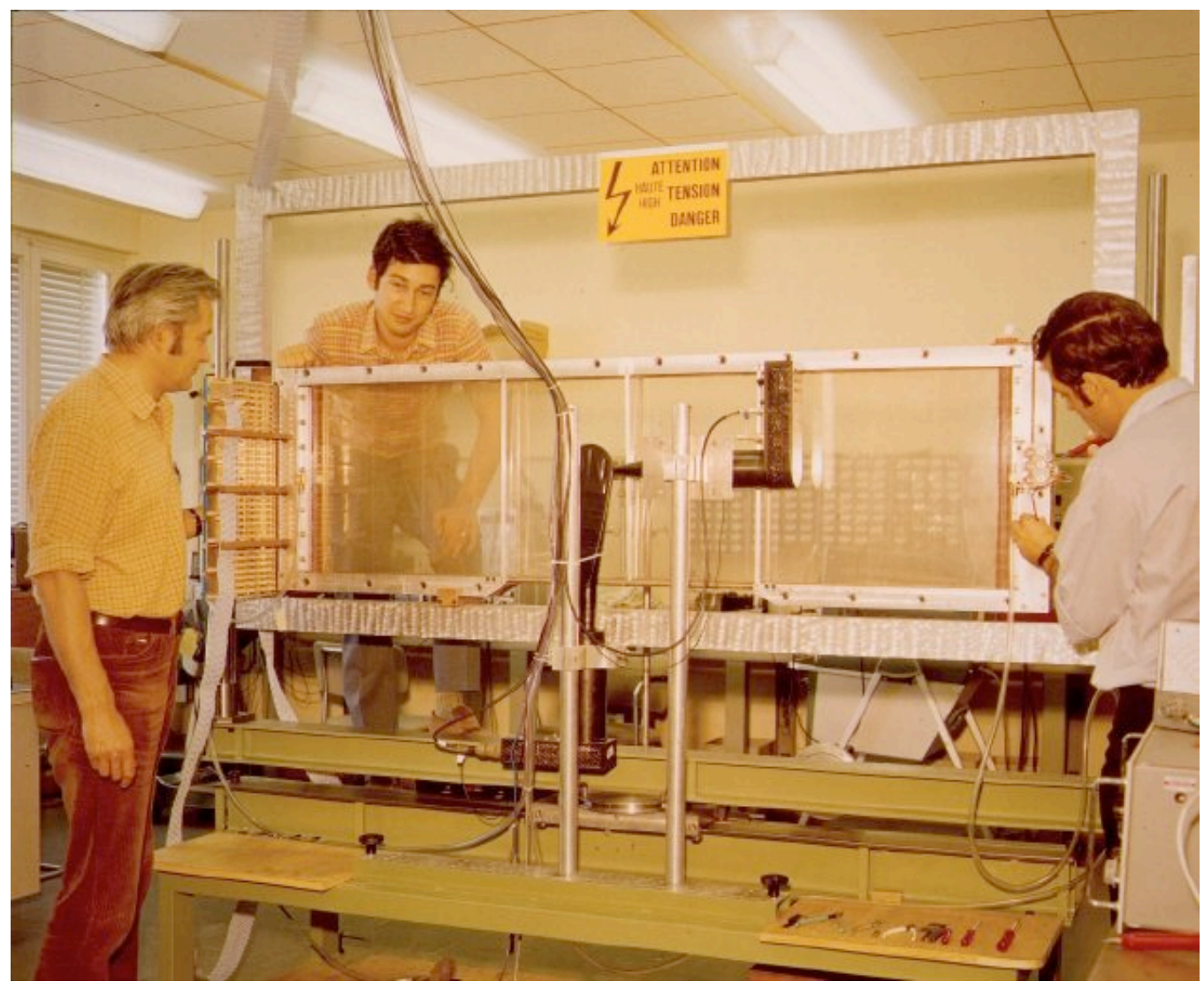

Fig. 4: A large MWPC prototype. Charpak stands at left, while the author overlooks the chamber in the centre.

For his invention of the multiwire chamber and the formidable impact of the new technology in experiments, G. Charpak was awarded the 1992 Nobel Prize in physics. 


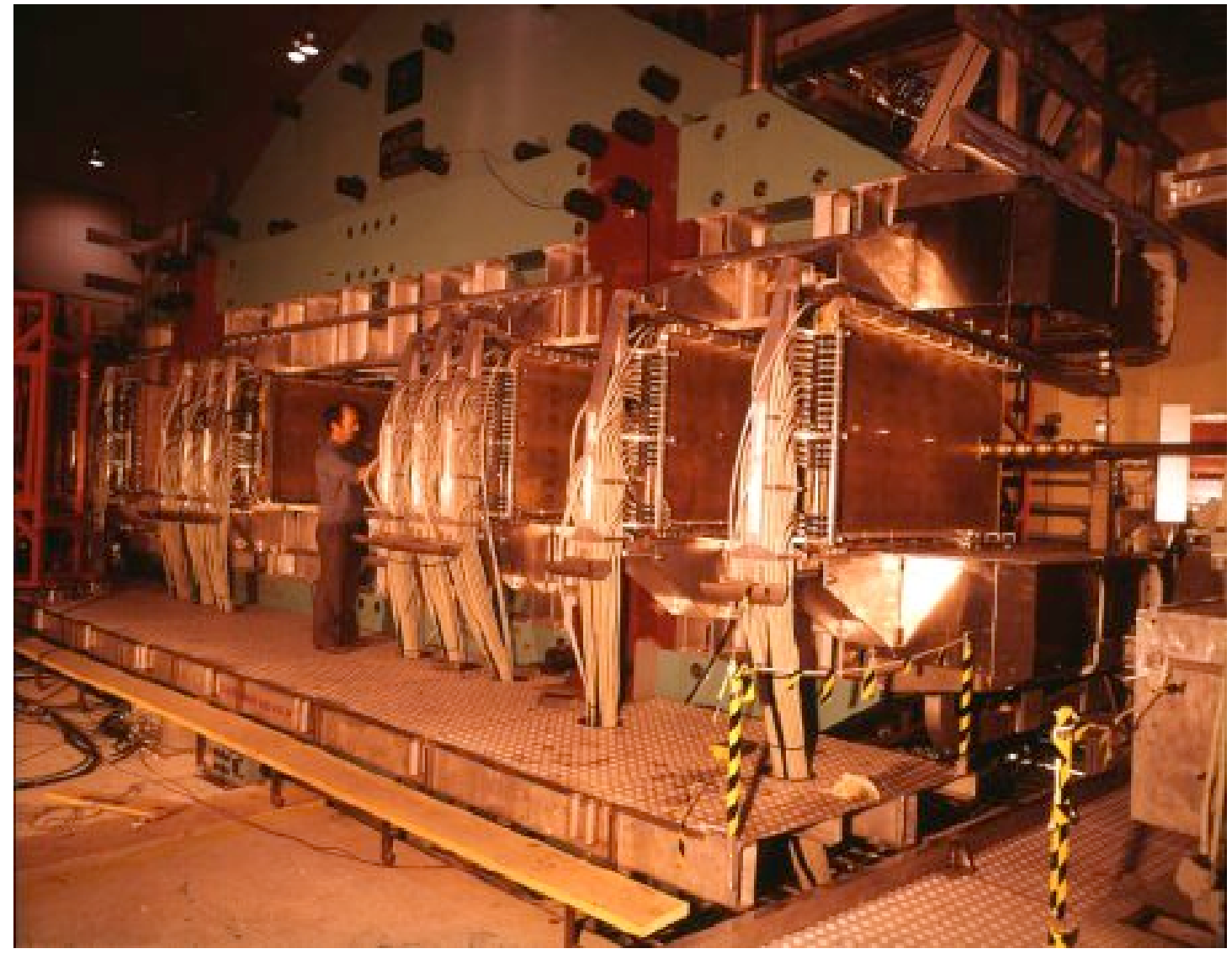

Fig. 5: The Split Field Magnet detector, mounting a set of large MWPCs.

A better understanding of basic gas phenomena and the exploitation of the information provided by the collection time of the released charge led to the conception of new families of wire detectors. In drift chambers, one records the time taken by electrons in wide gas cells to reach the anodes; space coordinates are deduced from a precise knowledge of the space-time correlation, determined by drift velocity, shape and strength of the applied electric field. Drift chambers were first used in an experiment at CERN in 1971 [10]. With simple multi-cell geometry, drift chambers can cover large detection areas, providing sub-millimetre accuracies with sense wire distances of tens of centimetres; this has permitted to build very large detectors at reasonable cost. Fig. 6 shows the experiment WA1, installed in CERN's neutrino beam from 1978. The set-up had alternate layers of magnetized iron, scintillation counters and hexagonal drift chambers, four meters in diameter, for position measurements [11]. Further downstream, another neutrino experiment (CHARM) used for localization a system of proportional and limited streamer tubes [12].

With a more sophisticated design, and a proper choice of the electric field structure and of the gas filling, drift chambers can achieve position accuracies better than $50 \mu \mathrm{m}$ [7]. These high-accuracy drift chambers have been used in many spectrometer-type set-ups, such as the channelling experiments at CERN and Fermilab, requiring very good space resolution. A pair of large high-accuracy drift chambers was in use for almost two decades in the Omega large acceptance spectrometer (Fig. 7). With improvements and modifications, drift chambers are widely used in most modern experiments; the long memory, determined by the drift time of all tracks belonging to an event, however limits their use to moderate rate environments. 


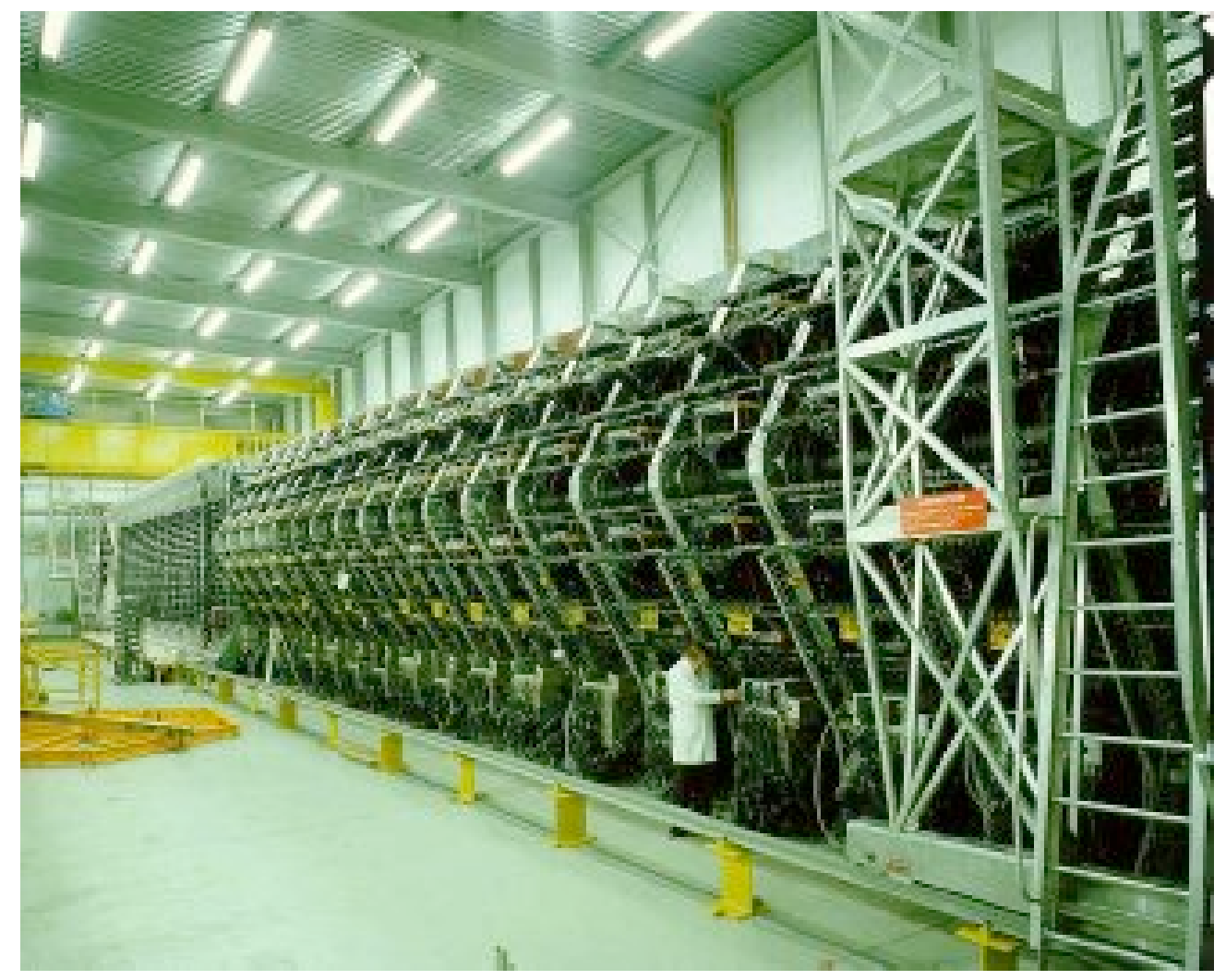

Fig. 6: The WA1 neutrino experiment, using large area wire drift chambers.

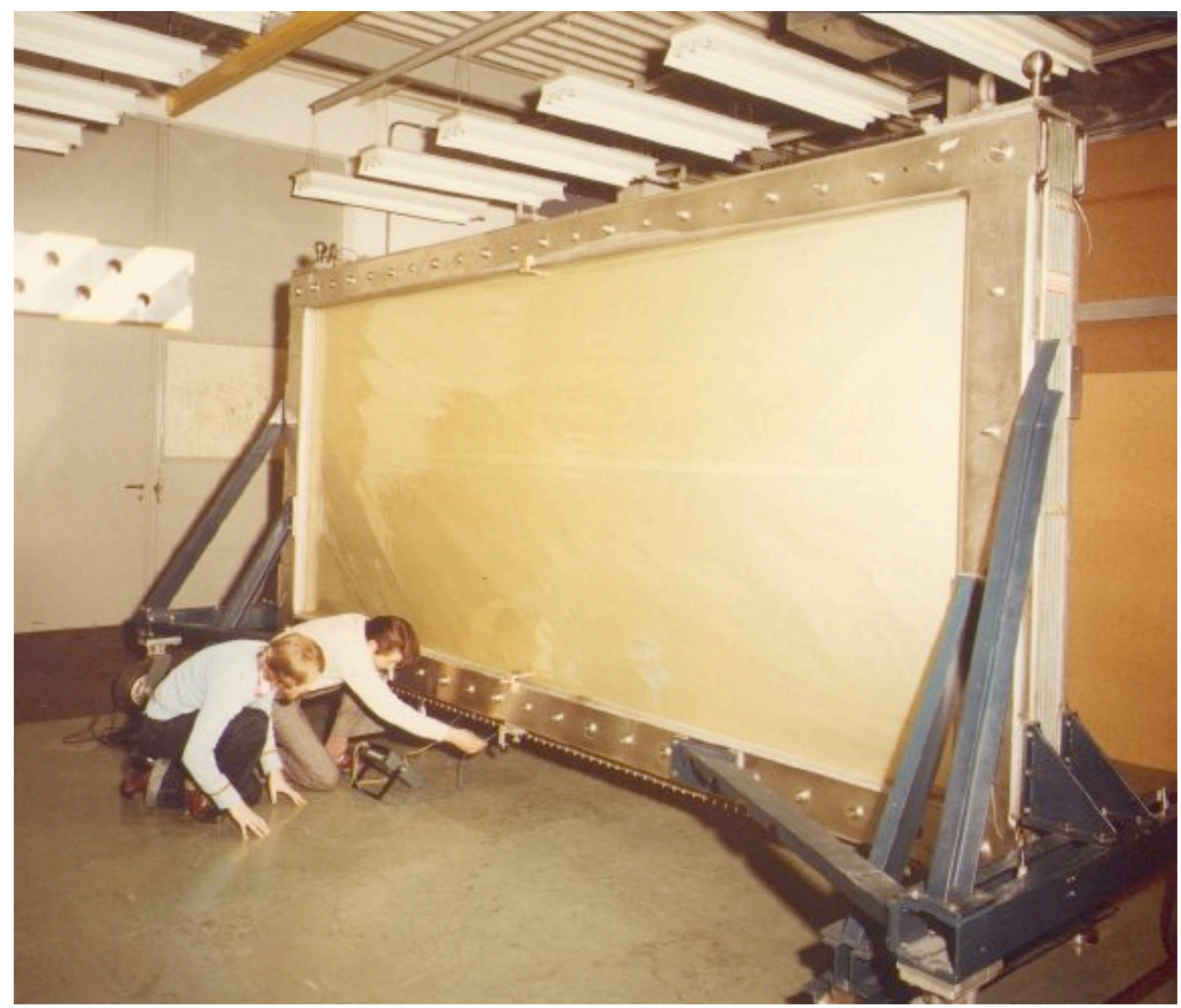

Fig. 7: A large High-Accuracy drift chamber for the Omega spectrometer. 
Perhaps the most powerful and performing gaseous detector is the Time Projection Chamber (TPC), introduced in the mid-seventies and first used in an experiment at SLAC [13]. It consists of a large gas volume, where ionising trails are produced by charged particles. A uniform electric field applied to the volume, between an upper cathode foil and a grid at the opposite end, drifts ionisation electrons towards the MWPC end-cap detector; smoothness of drift is guaranteed by the uniformity of the electric field, achieved with the help of graded potential rings placed all around the cylinder, the so-called field cage. The end-cap MWPC itself has a set of anode wires to collect and amplify the drifting charge, and an outer cathode with a pattern of strips or pads used to record the induced charge profiles, sampled in contiguous time slices (hence the name). Usually cylindrical in shape to fit inside a solenoid, two TPCs can be mounted back-to-back, symmetrically around the interaction region.

\section{THE PROTON-ANTIPROTON COLLIDER EXPERIMENTS}

A formidable research effort was undertaken to improve the pattern recognition and particle identification capabilities of electronic devices, bringing their performance close to the ones of bubble and streamer chambers. This goal cannot be achieved with a monolithic detector: modern systems consist of a succession of modules serving different functions in sequence, in an onion-like multi-shell structure. A meticulous design is needed to cover the solid angle, avoiding blind regions or cracks in the setup, a property called hermeticity. One of the first examples of hermetic detectors is the complex built for the UA1 experiment at CERN's proton-antiproton collider, a reference for many later devices [14]. A system of drift chamber modules, surrounding the central vacuum tube and filling the central part of the magnet constituted the main tracker; the modules are mounted with different orientations in the central and forward regions, to optimize the response (Fig. 8) [15]. Particle identification was achieved in a sequence of detectors: a lead-scintillator electro-magnetic calorimeter inside the magnet, an external ironscintillator hadronic calorimeter and a muon identifier making use of large area drift chambers and drift tubes. Fig. 9 shows the installation of the central detector between the two open poles of the magnet. Innovative technologies, nowadays standard in experiments, were developed by the UA1 group: a drift chambers calibration system exploiting straight tracks produced by miniature ultra-violet laser beams, the use of early personal computers for the experiment control, novel on-line colour event displays. Fig. 10 is an example of the imaging capability of the detector.

Equally innovative, the second $p-\bar{p}$ experiment (UA2) made extensive use of multi-wire and drift chambers for the detection of the interaction vertex as well as for electro-magnetic and hadronic calorimetry. The two experiments started taking data in 1981, and led to the experimental discovery of the neutral and charged massive vector bosons. Capital for the realization of the experiment was the development of stochastic beam cooling, reducing the momentum spread of the antiprotons at the production, thus permitting to reach the desired accumulated beam intensities.

The physics discovery and technological achievements of the $p-\bar{p}$ experiments were recognized by the attribution in 1984 of the Nobel prize in physics jointly to Carlo Rubbia and Simon van der Meer. 


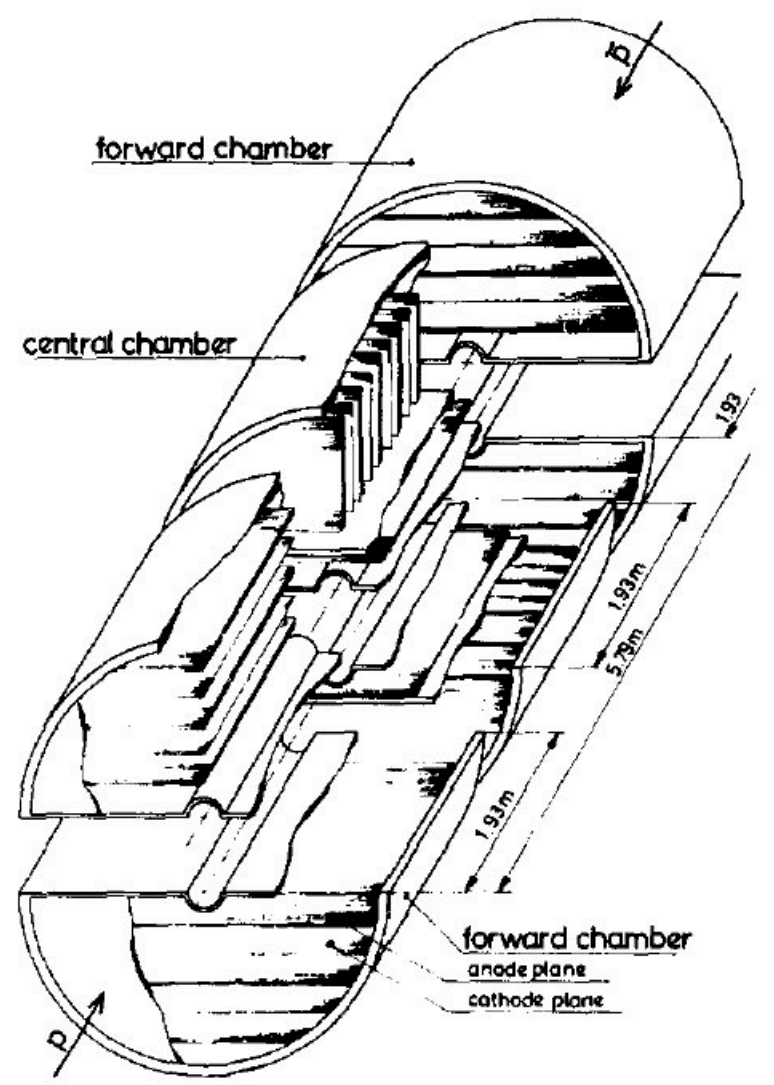

Fig. 8: Modular drift chambers system for the UA1 experiment.

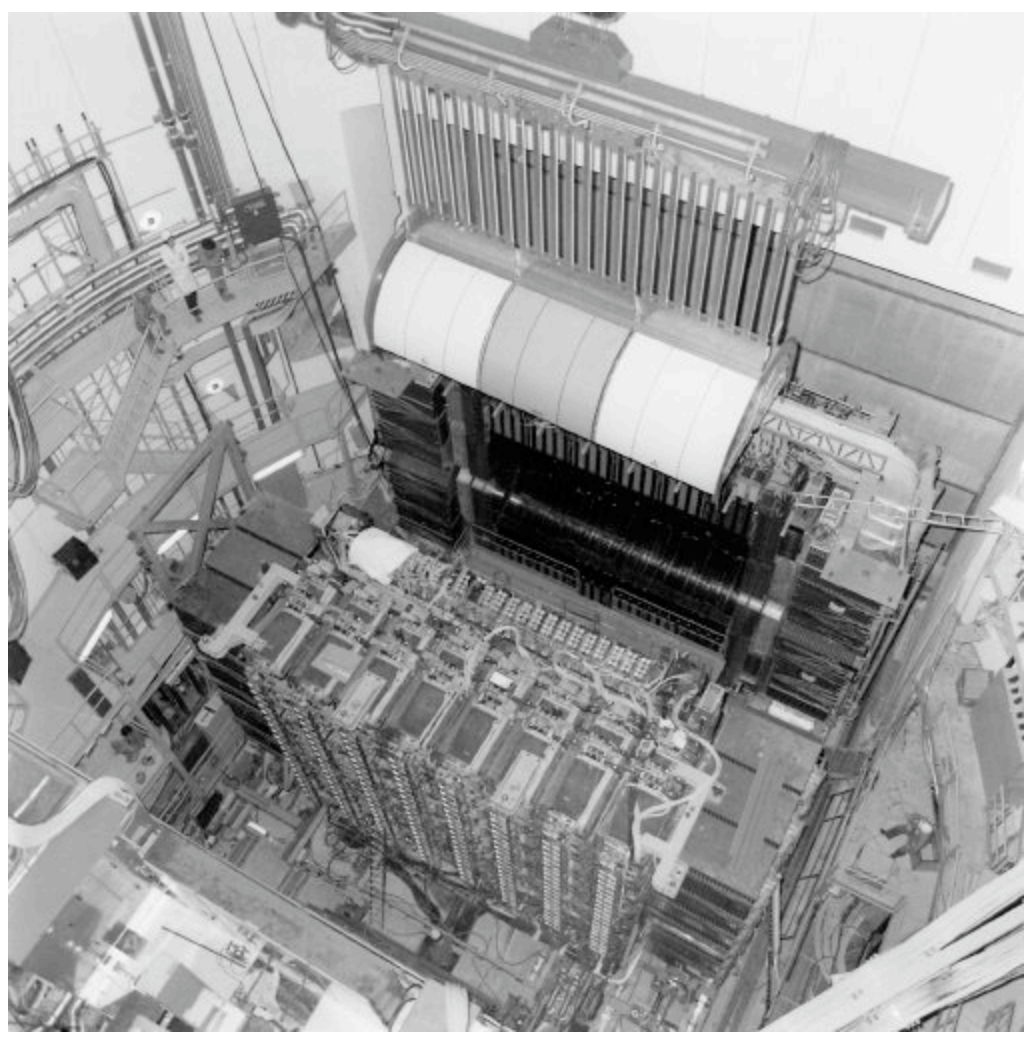

Fig. 9: View into the UA1 set-up, open for maintenance. The upper half of the drift chamber tracker is being lowered within the magnet poles. 


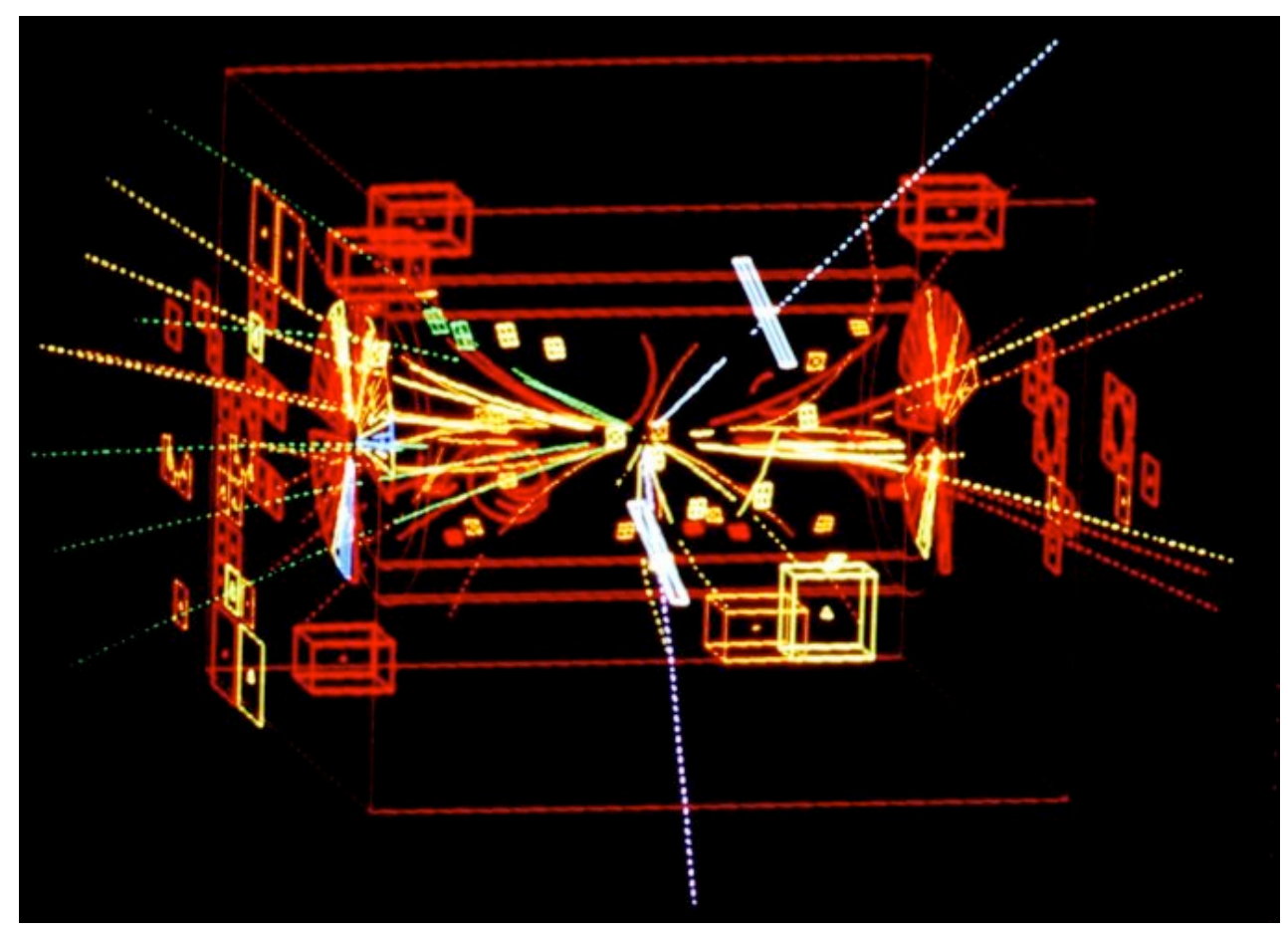

Fig. 10: Computer display of the first $Z_{0}$ decay observed in UA1.

\section{THE DAWN OF SOLID STATE DETECTORS}

Despite many improvements, position-sensitive detectors based on wire structures are limited by basic diffusion processes in the gas to localization accuracies around a tenth of a mm. A more performing tool became available in the eighties with the introduction of the first silicon micro-strips. The detectors are manufactured on thin silicon wafers, on which individual junction diodes shaped as parallel strips are realized combining the techniques of oxide passivation, photolithographic engraving and ion implantation (Fig. 11). Typically $300 \mu \mathrm{m}$ thick, the diodes can be fully depleted by the application of a reverse voltage, and act as individual ionisation detectors. They provide space coordinates with microns accuracy using a high density of strips, or indirectly recording the charge shared between adjacent strips. Fig. 12 shows one of the early silicon micro-strip detectors, developed in the early eighties; the sensor had an active area of $24 \times 36 \mathrm{~mm}^{2}$, with a much larger volume taken by the readout electronics [16]. With improvements, detectors of similar design were used to tag charmed particle decays in the NA11/NA32 spectrometer at CERN.

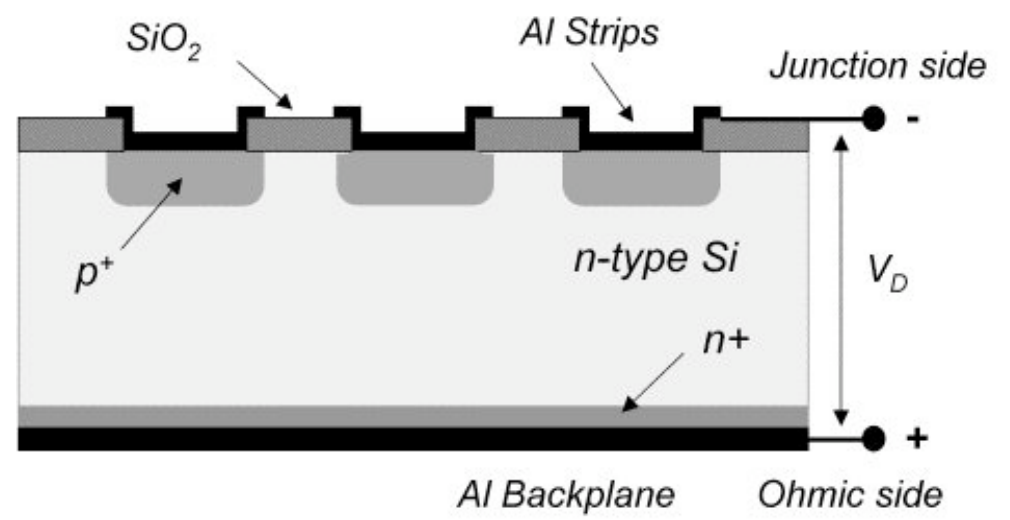

Fig. 11: Schematics of a silicon micro-strip detector. 


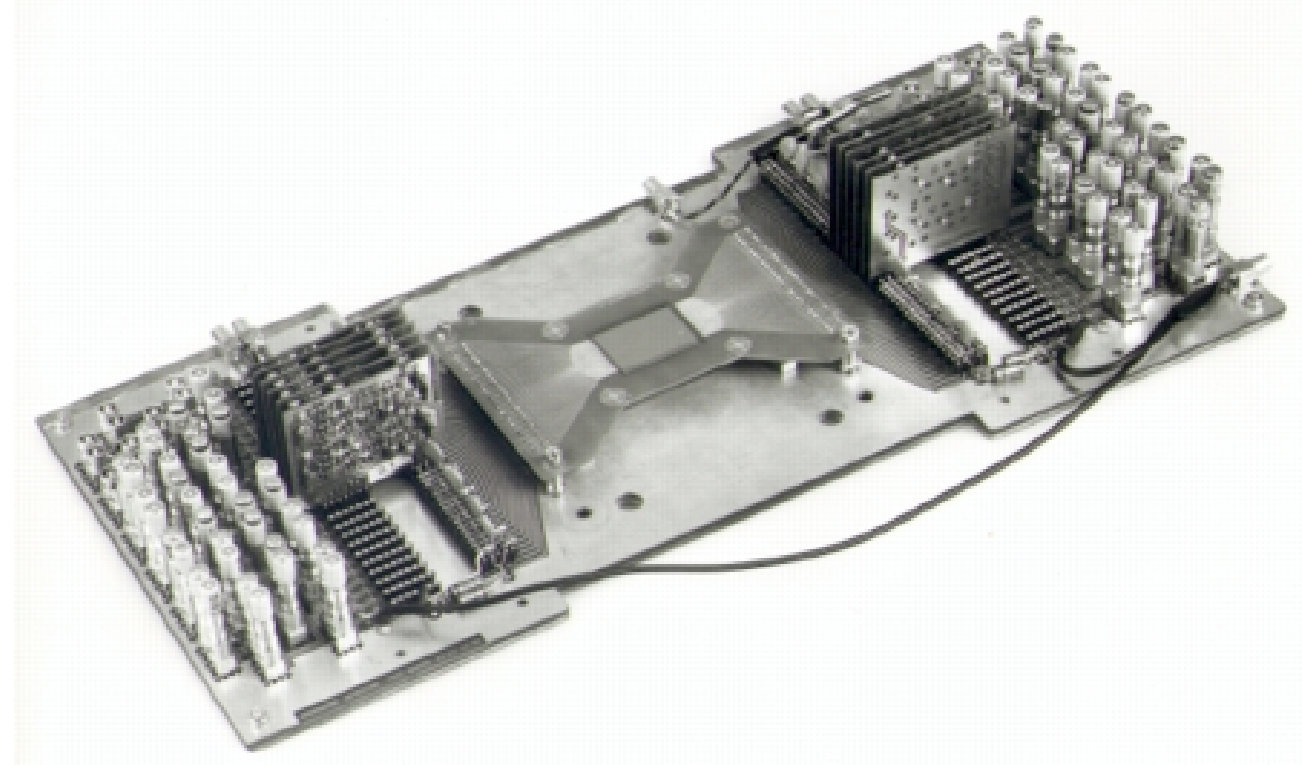

Fig. 12: One of the first silicon micro-strip detectors, developed for experiment NA11. The sensor had 100 strips at $200 \mu \mathrm{m}$ pitch.

Silicon micro-strip detectors gained a gradually increasing role in particle physics, covering larger and larger areas, thanks also to the concurrent development of low cost, highly integrated electronics capable of recording the charge released in the thin silicon layer. Already in the mid-eighties, high-density circuits manufactured with nMOS and CMOS technology were developed, able of handling a large number of analogue input signals multiplexing the information on a single output line. Fig. 13 shows the AMPLEX chip, a 16-channels CMOS low-noise, low power analogue signal processor used in the UA2 experiment [17]. Reference [18] gives a comprehensive review of the status of the technology in the mid-eighties.

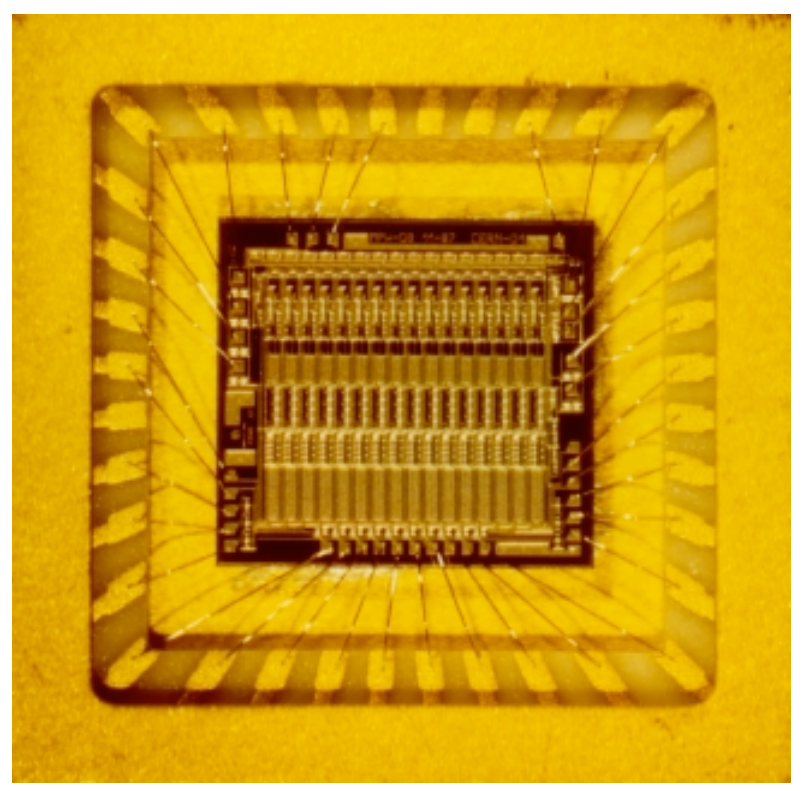

FIG. 13: AMPLEX, a 16-channels analogue multiplexer. 


\section{DETECTOR DEVELOPMENTS IN THE EIGHTIES}

Many innovative detectors were developed in the eighties, particularly significant for the progress in particle identification and calorimetry. In TPC-like devices, a detailed study of the statistics of energy loss and a thorough choice of the gas fillings permitted to improve the resolution in energy loss measurements, providing information on the particle velocity; this is a very powerful tool for mass identification, particularly in the relativistic rise region. Designed to achieve a better multi-track resolution, the Time Expansion Chamber (TEC), employs a gas with slow drift velocity and very low diffusion to bring to the wire amplifying structure a "slow motion" image of the ionising trails, thus exploiting at best the timing characteristics of the electronics [19]. Fig. 14 is a close-up of the wire structure in the TEC built for the L3 experiment at LEP.

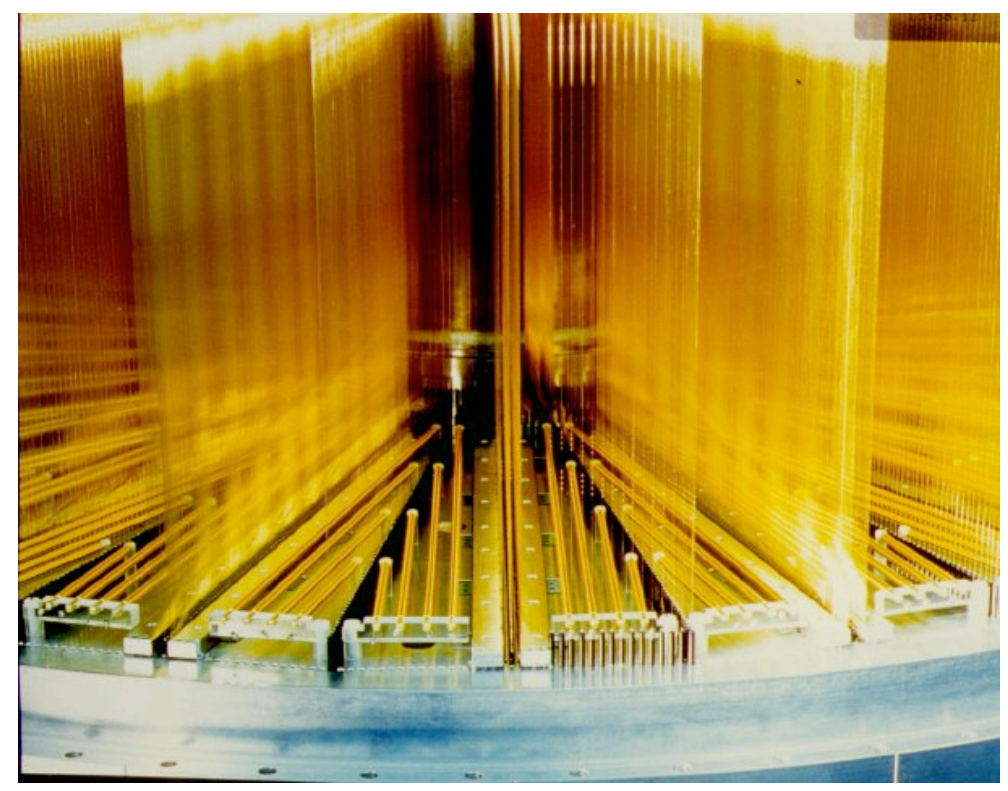

Fig. 14: Close view of the wire planes in the Time Expansion Chamber.

Photons emitted by Cherenkov effect can be focussed with suitable optics in a ring pattern, whose diameter is a function of the particle velocity. In 1977, Thomas Ypsilantis and Jacques Seguinot developed a multiwire proportional chamber, filled with photosensitive vapours, to detect these photons in the ultra-violet domain [20]. Originally named CRID (Cherenkov Ring Imaging Detector), the new device is now better known as RICH; reference [21] provides a good historical survey of the ring imaging technology and applications. Large gas gains are required for the detection and localization of single photoelectrons; this is hard to achieve in photosensitive gases, due the feedback generated by copious photon emission in the large avalanches. Introduced by Charpak and Sauli in 1978, the Multi-Step Chamber permitted to solve the problem [22]. The device combines in the same detector a parallel plate pre-amplification stage and a conventional MWPC. Sharing the gain between two stages, larger amplifications can be obtained before feedback processes set in, achieving full detection efficiency of single photoelectrons. The coordinates of the photons can be reconstructed unambiguously recording the detected charge on anode and cathode wires, placed at angles to each other, or with a matrix of small pads covering one of the cathodes. A large acceptance RICH making use of multistep chambers, built by a CERN-Saclay-Stony Brook collaboration, has been operational for many years in the experiment E605 at Fermilab [23]. 
The first generation $\mathrm{RICH}$ detectors used photosensitive vapours having relatively high photo-ionization thresholds; this restricts detection to vacuum ultraviolet photons, and requires the use of fragile and expensive fluoride windows. A substantial progress was finding a vapour, TMAE, having a much lower threshold thus permitting to utilize quartz windows [24]. A TMAE-filled multi-step RICH with pad readout has been used in the early nineties by the experiment NA45/CERES; Fig. 15 shows an example of recorded event [25].

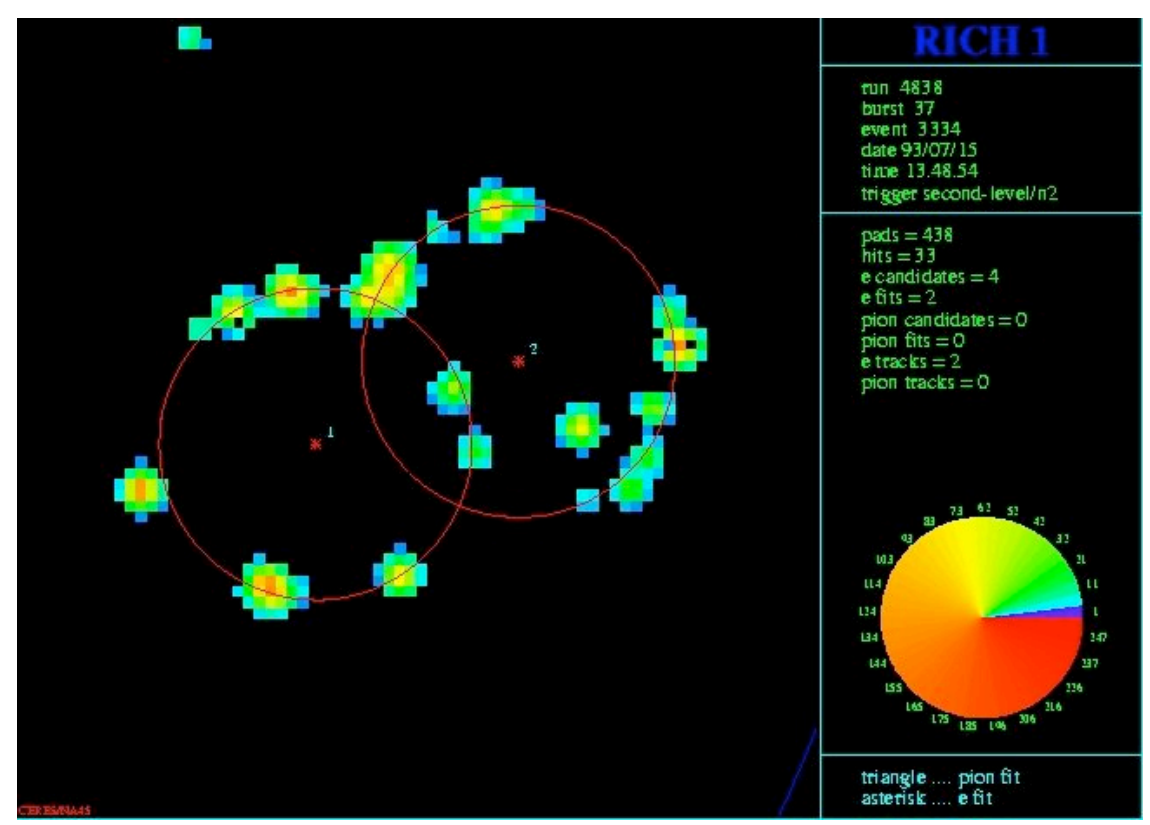

Fig. 15: A two-ring event recorder with the CERES RICH. The detector is a multi-step proportional chamber with pad readout.

A RICH design using drift chambers as photon detectors permits to decrease the number of readout channels required, at the expense of a reduced rate capability. It consists of a long, thin drift volume, delimited by UV-transparent quartz windows with field-shaping wires, guiding the photoelectrons to a small end-cap MWPC [26]. To increase the velocity range of particles that can be identified, the same drift chamber can be coupled on one side to a gas radiator, with mirrors reflecting the photons in a ring pattern, and on the other to a liquid radiator with rings forming directly by proximity focussing. Fig. 16 shows schematically a prototype built for the DELPHI experiment. A system of RICH detectors with the drift chamber design has been operating in the Omega spectrometer between 1984 and 1994; with various upgrades, it has significantly contributed to the physics results of the experiments [27].

The high-density drift chamber (also known as HPC, High density Projection Chamber), originally developed to detect high-energy photons in view of medical applications [28], has been adapted to serve the role of electromagnetic calorimeter. In the detector, the gaseous drift volume is filled with a stack of heavy metal converters, separated by a dielectric and with open channels. Ionisation electrons, released in the open channels by radiation interacting in the converters, drift under the effect of an applied field until reaching the end-cap proportional chamber used for detection. In preparation for the use in DELPHI, a large effort was put in optimising its large scale manufacturing and test procedures [29]. As shown in Fig. 17 , the foil converters have been replaced by lead wire, woven around insulating supports, leaving open channels for the ionization production and drift. 


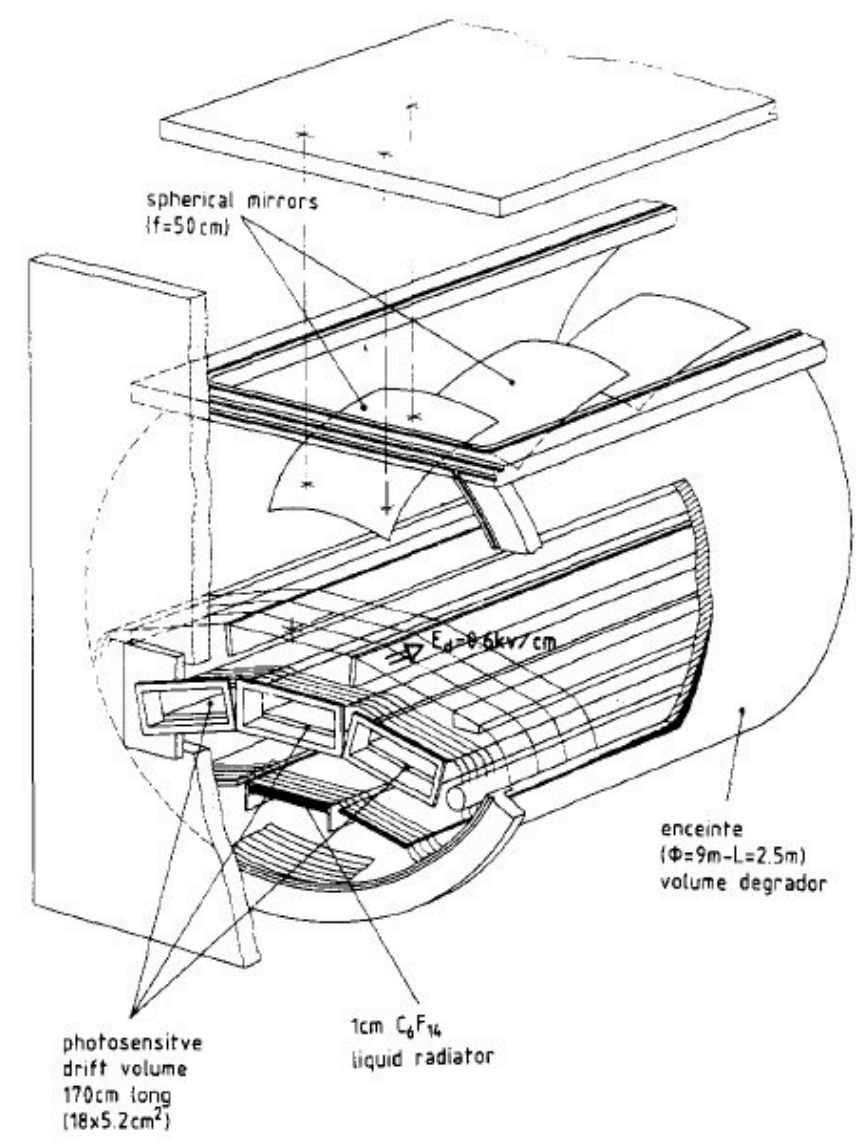

Fig. 16: Prototype of the DELPHI Barrel RICH detector.

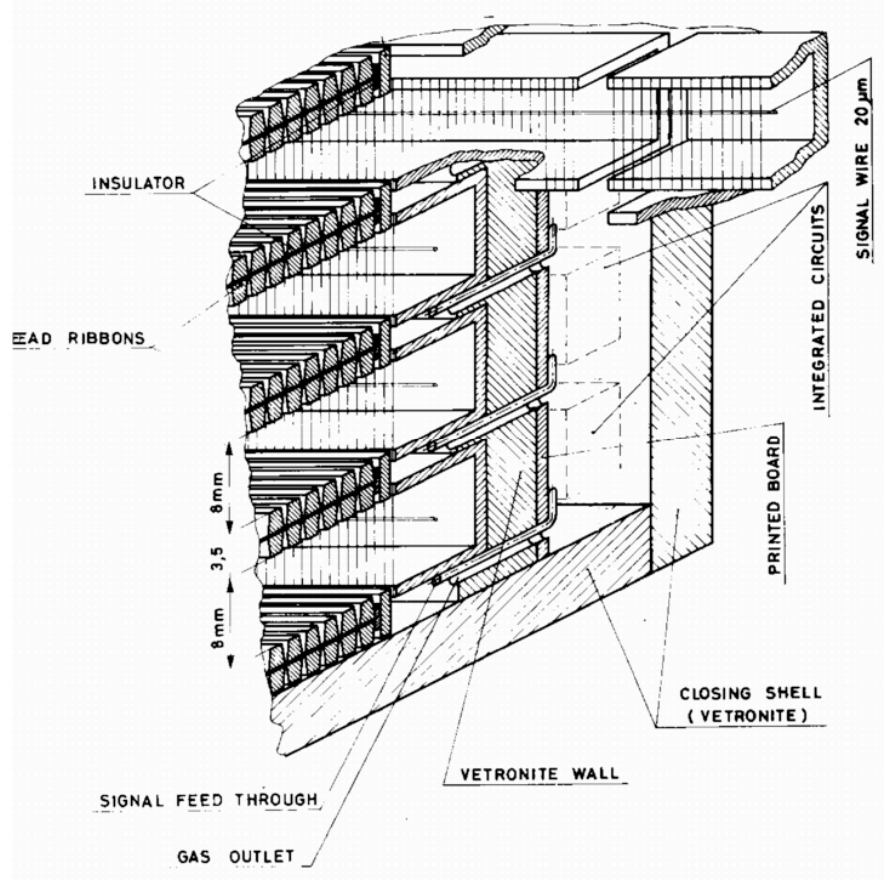

Fig. 17: High Density Projection Chamber: view of the drift region with lead wire converter layers. 
Plastic scintillation counters are widely used for triggering and calorimetry; the conventional way of extracting the scintillation signals with plastic light guides is however rather inconvenient for large systems. The development of wavelength shifter (WLS) bars, optically coupled to the scintillators, simplified to a great extent the assemblies. The technology evolved later with the use of thin WLS fibres that could be inserted in the scintillator slabs. A survey on the use of scintillators, as well as other technologies in calorimetry in the eighties can be found in Ref. [30] .

A large collaborative effort has been invested in the development of new scintillators, having high density, large light yield and a fast response; a large attenuation length and high radiation resistance add to the requirements. The best candidates were found in the class of inorganic crystals, such as bismuth germanate $\mathrm{Bi}_{4} \mathrm{Ge}_{3} \mathrm{O}_{12}$ (BGO), barium and cerium fluoride $\left(\mathrm{BaF}_{2}, \mathrm{CeF}_{3}\right)$. With its short radiation length and high density, $\mathrm{BGO}$ was the best candidate for the construction of compact electromagnetic calorimeters [31]. Its relatively long scintillation decay time restricts however the use for moderate rate experiments, a good motivation for continuing the quest of alternatives.

\section{GENERAL PURPOSE DETECTORS AT LEP}

A major impetus to the development and exploitation of detectors came with the construction of LEP, CERN's electron-positron collider, commissioned in 1989. The rapidity range to be covered and the requirements of hermeticity led to the concept of general-purpose magnetic detectors with good tracking and particle identification properties. With the increase of the centre of mass energy in the collision, short-lived particles have a perceptible range before decaying, and an essential aspect of event reconstruction is the proper identification of secondary vertices. This has been accomplished exploiting the precision and multi-track resolution power of solid state devices, combined to the pattern recognition capability of large volume gaseous tracking detectors.

Four large set-ups have been built and operated: they shared common features, such as hermeticity and precise vertex tracking, as well as an accurate momentum analysis of secondary tracks with a solenoidal magnetic field surrounding the core of the experiment. For better coverage, with a geometry that became standard, the various components are grouped in two sub-systems: a cylindrical assembly covering large to intermediate angles (the barrel), and forward parts perpendicular to the beam axis, covering the small angle regions (the end-caps).

Fig. 18 shows a general view of DELPHI (DEtector with Lepton, Photon and Hadron Identification [32]. A large solenoid, $3 \mathrm{~m}$ in diameter, contained in sequence a silicon vertex detector, an inner detector made with wire chambers, and a Time Projection Chamber; their combined use provided trigger selection, charged track reconstruction and momentum analysis. Originally consisting of two layers of silicon strips, the vertex detector underwent several upgrades, with the addition of an inner layer and later a readout of the coordinate along the beam (Fig. 19). Next to the TPC, a Ring Imaging Cherenkov detector (RICH) added a powerful particle identification capability. Fig. 20) shows the RICH barrel, consisting of a set of photon-sensitive drift chambers, being inserted into the mirror assembly. Still inside the coil, High density Projection Chambers served as electromagnetic calorimeter; a set of limited streamer chambers in the iron return yoke and large size muon drift chambers completed the setup. . Fig. 21 gives an example of a reconstructed event. 


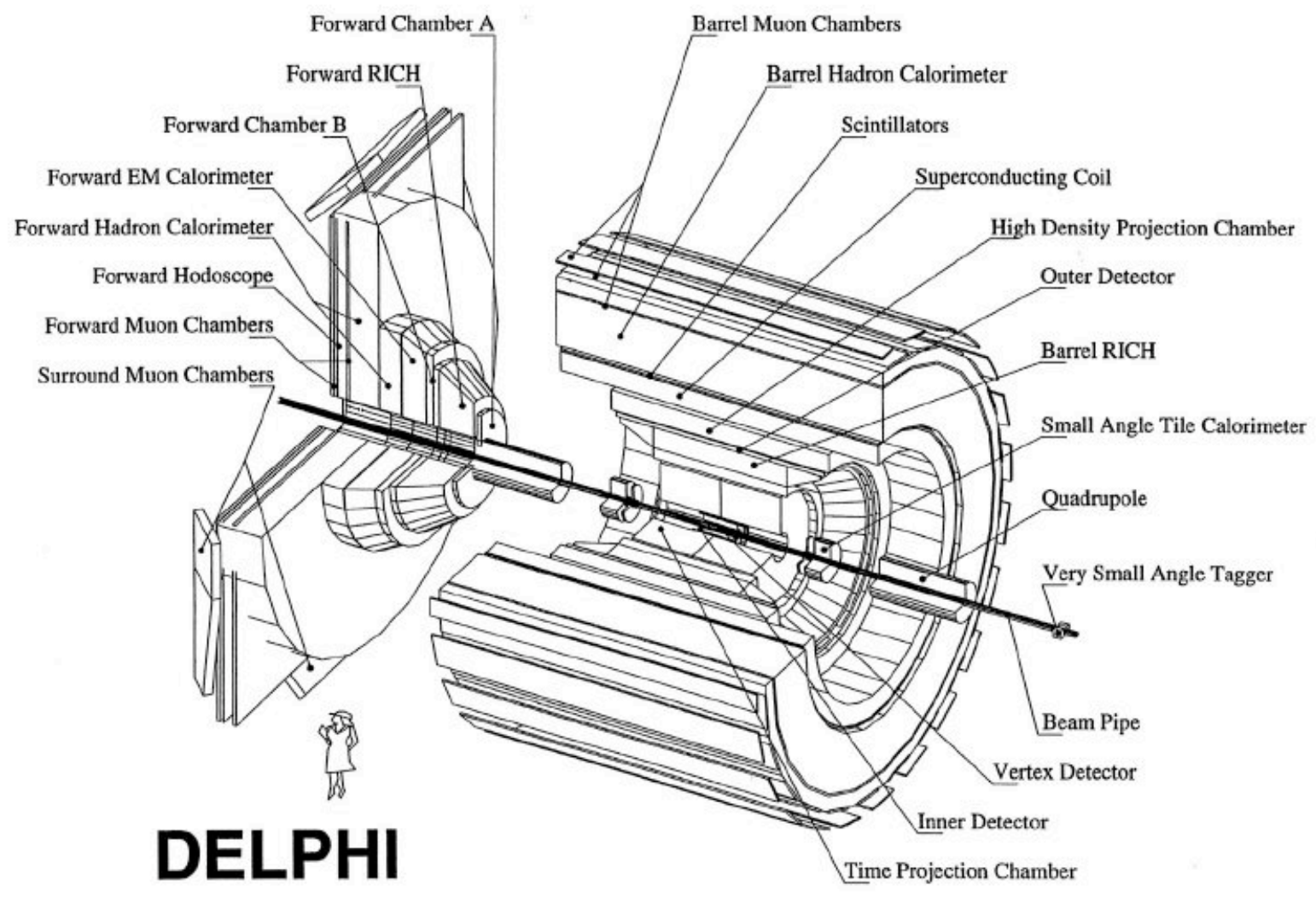

Fig. 18: Schematics of the DELPHI experiment.

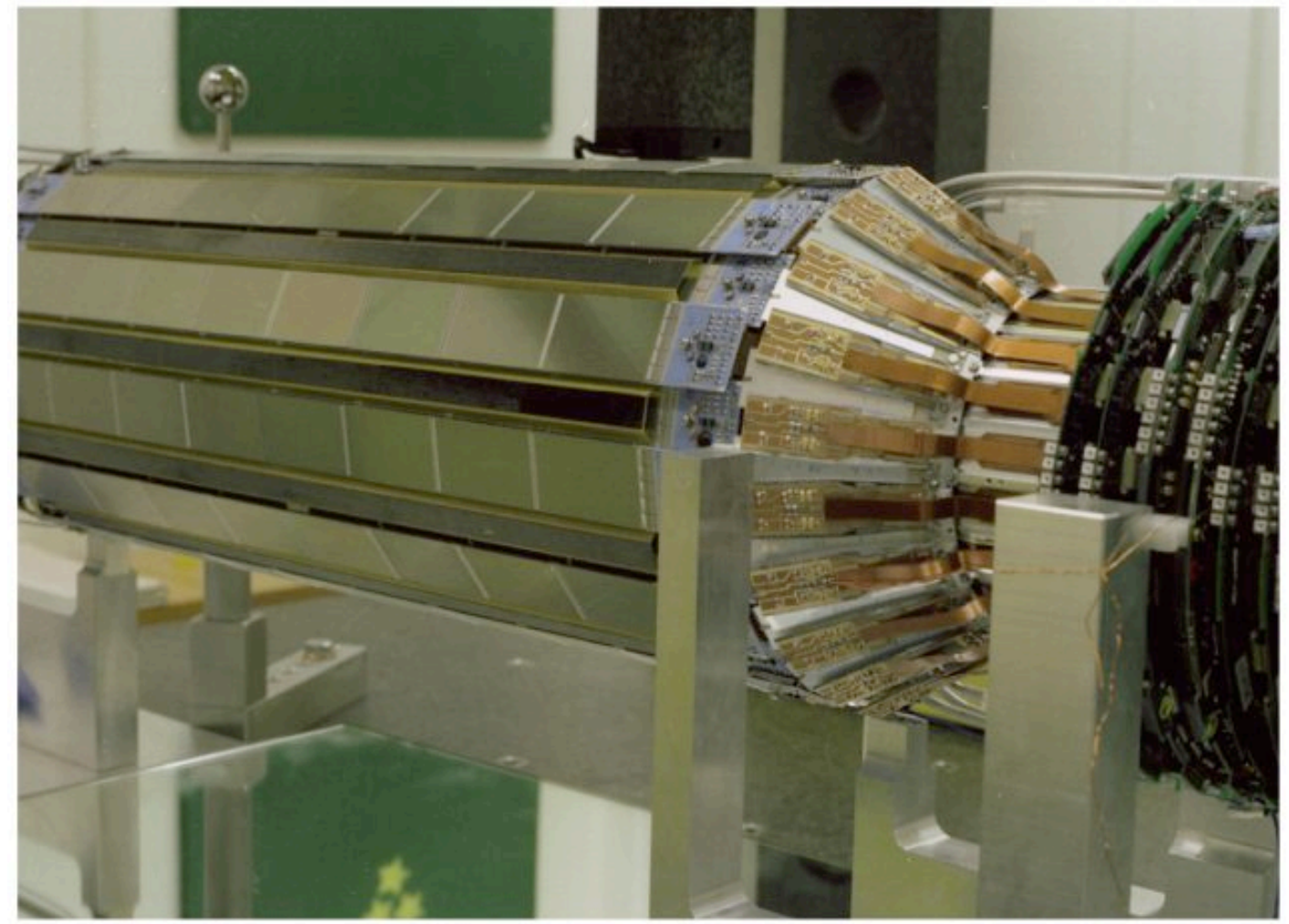

Fig. 19: The DELPHI silicon micro-strip tracker. 


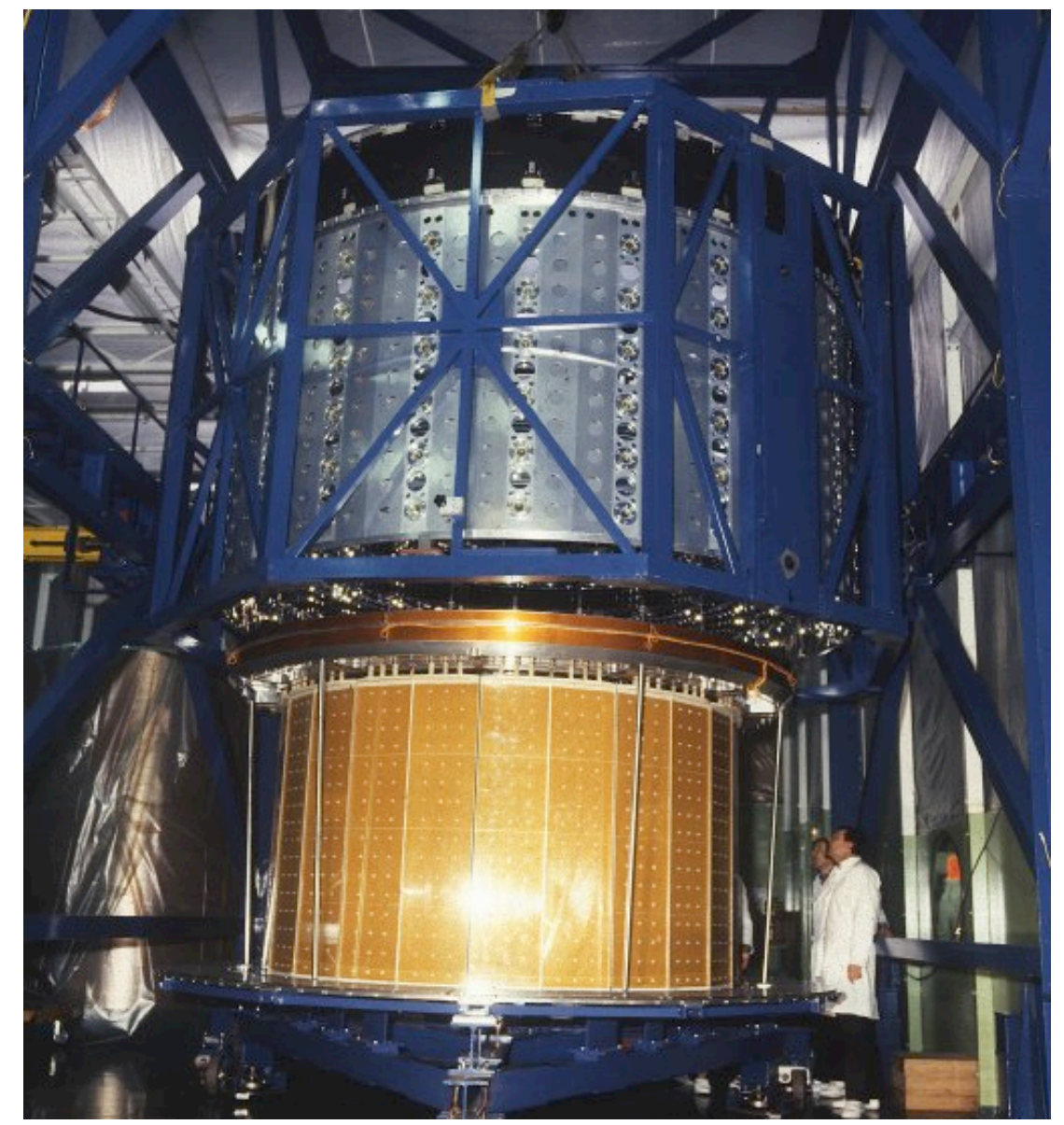

Fig. 20: DELPHI RICH detector being inserted into the mirror's barrel.

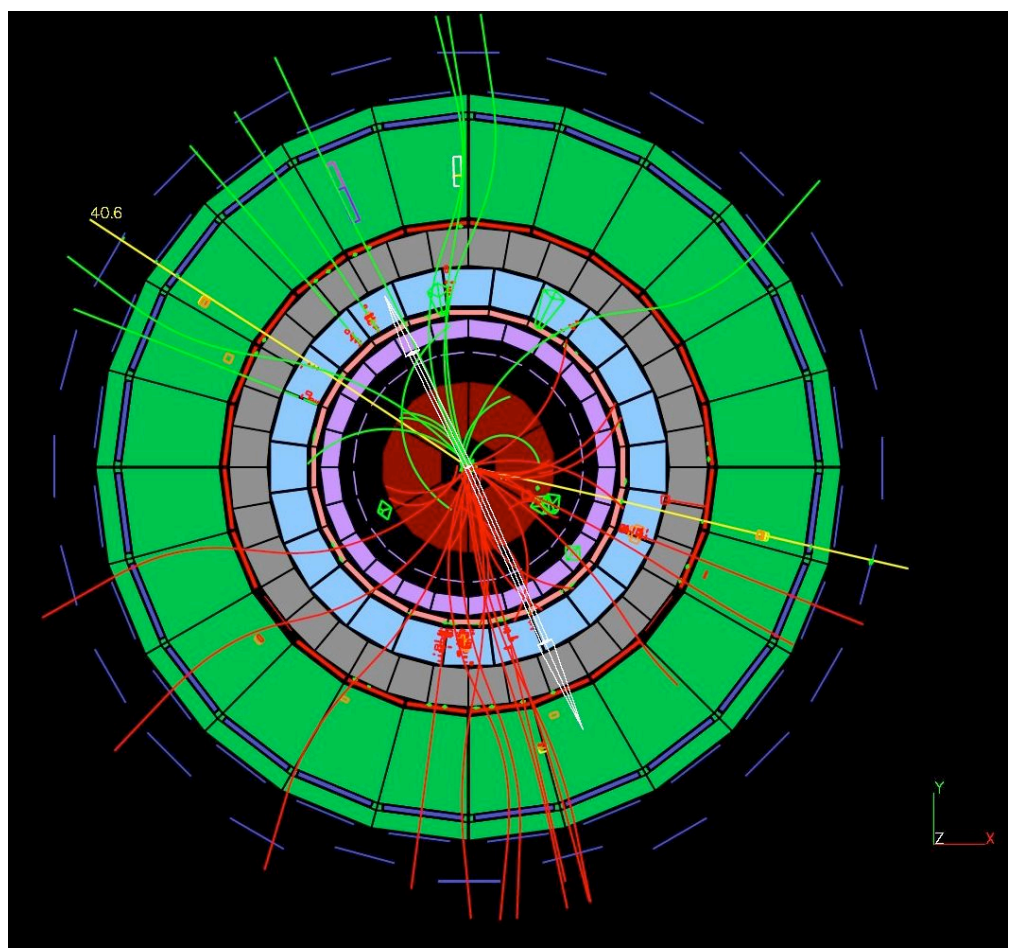

Fig. 21: Event display in DELPHI. 
Similar in conception, ALEPH (Fig. 22) [33] had a set of silicon microstrip detectors arranged in coaxial cylinders with contiguous, partly overlapping ladders. In a 1995 upgrade, the length of the detector was increased and the radial resolution improved using six double-sided detectors per ladder (Fig. 23). The excellent tracking performance permitted to identify secondary vertices due to the decay, within the vacuum tube, of short-lived particles. A drift chamber with $30 \mathrm{~cm}$ outer radius, important part of the trigger selection, and a large TPC (Fig. 24) completed the central tracking system. Electromagnetic calorimetry was performed with a leadMWPC sandwich, followed by an iron-streamer tube hadron calorimeter. Fig. 25 is a front view of the detector; the spokesman of the experiment and Nobel laureate Jack Steinberger stands third from left.

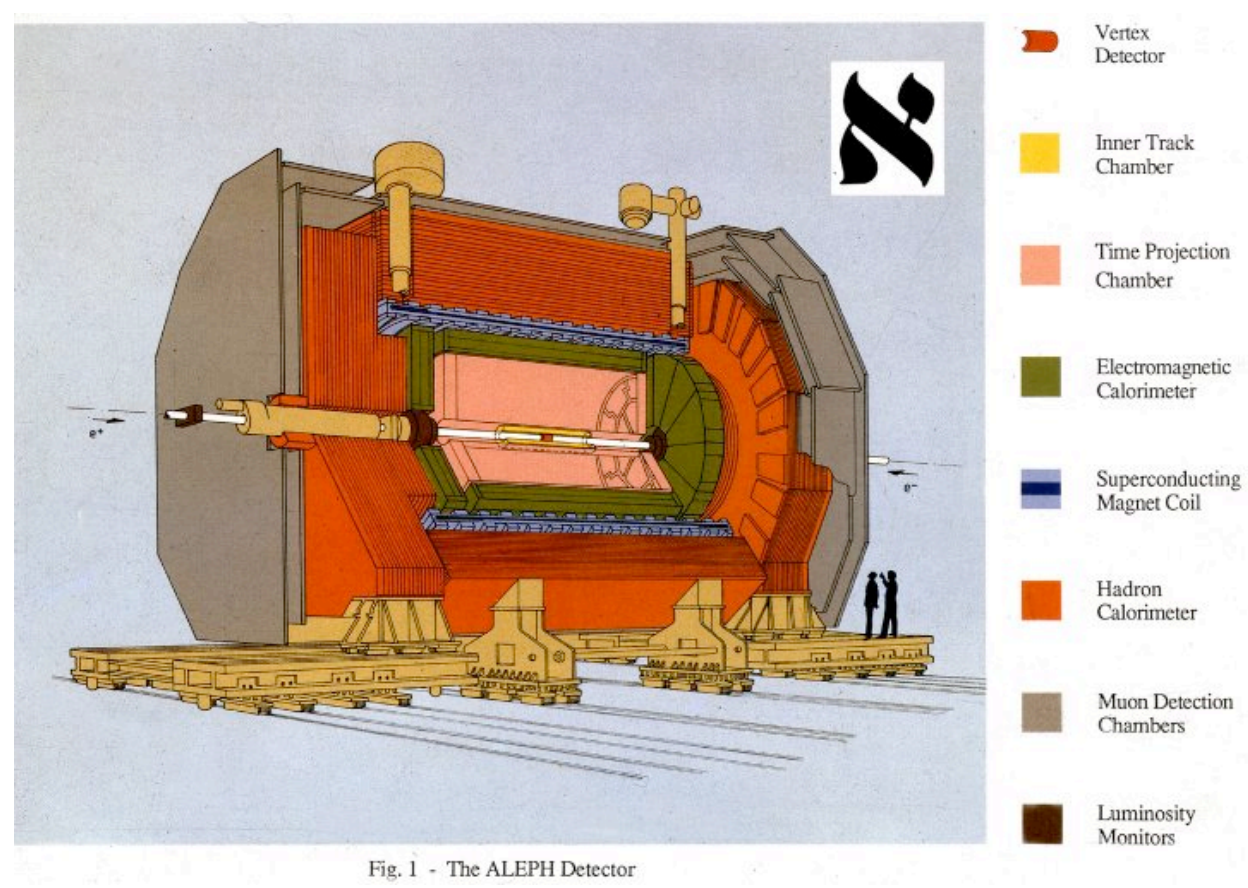

Fig. 22: The ALEPH detector.

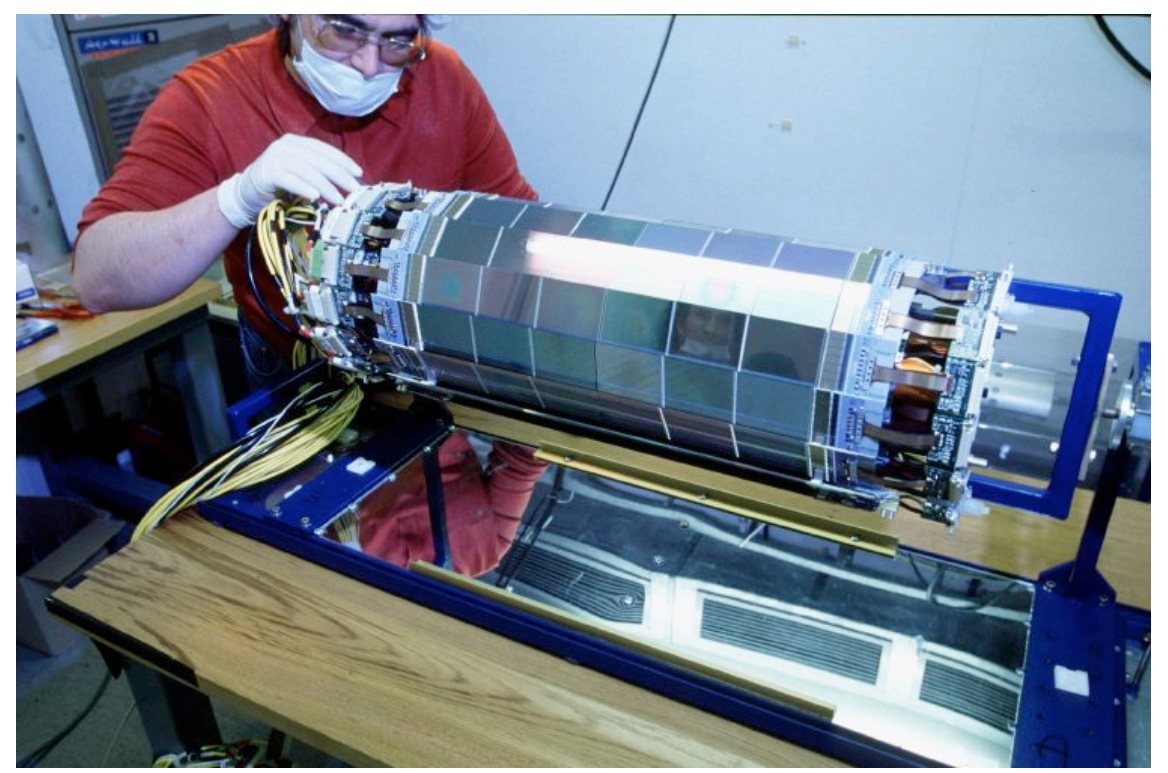

Fig. 23: ALEPH upgraded silicon micro-strip vertex detector. 


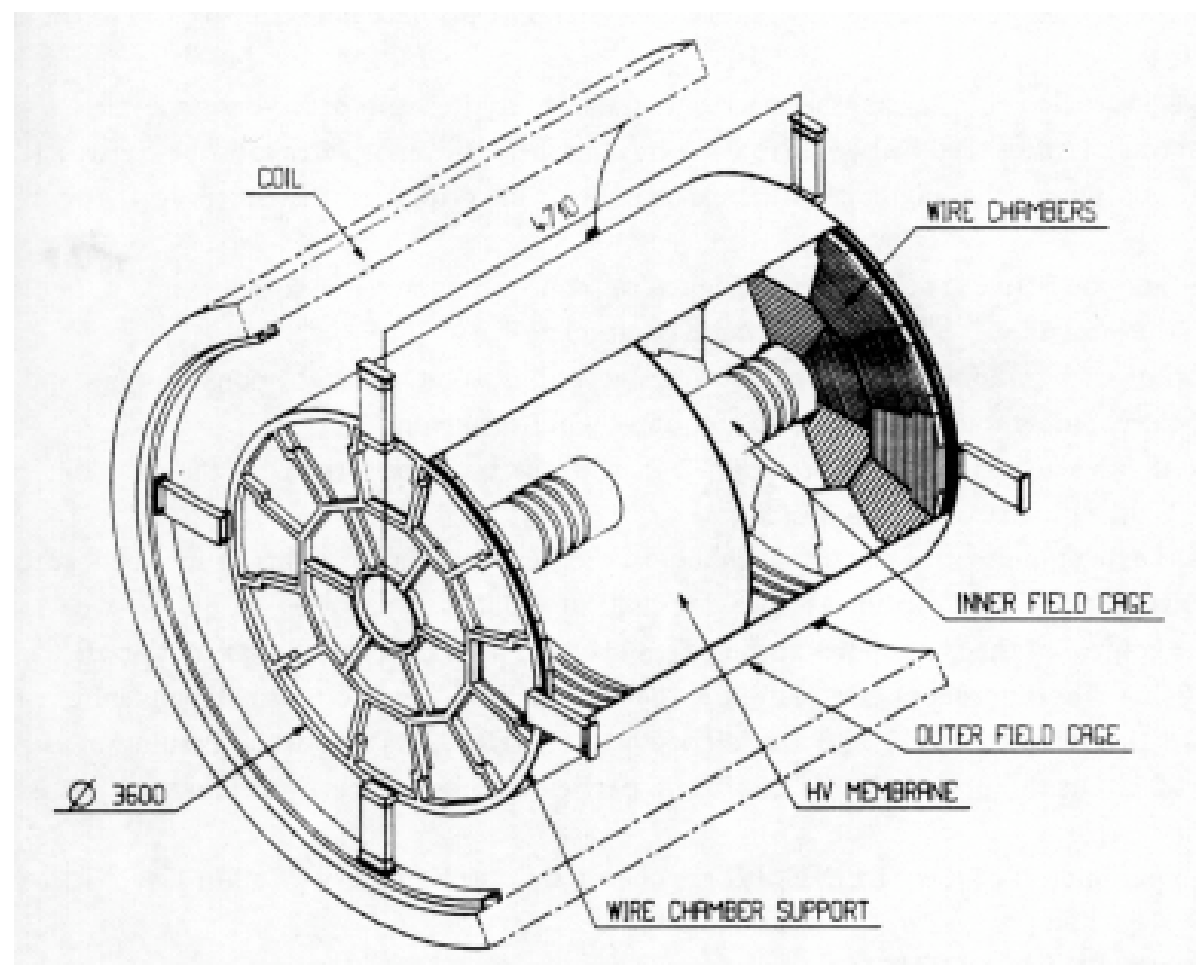

Fig. 24: Schematics of the ALEPH Time Projection Chamber.

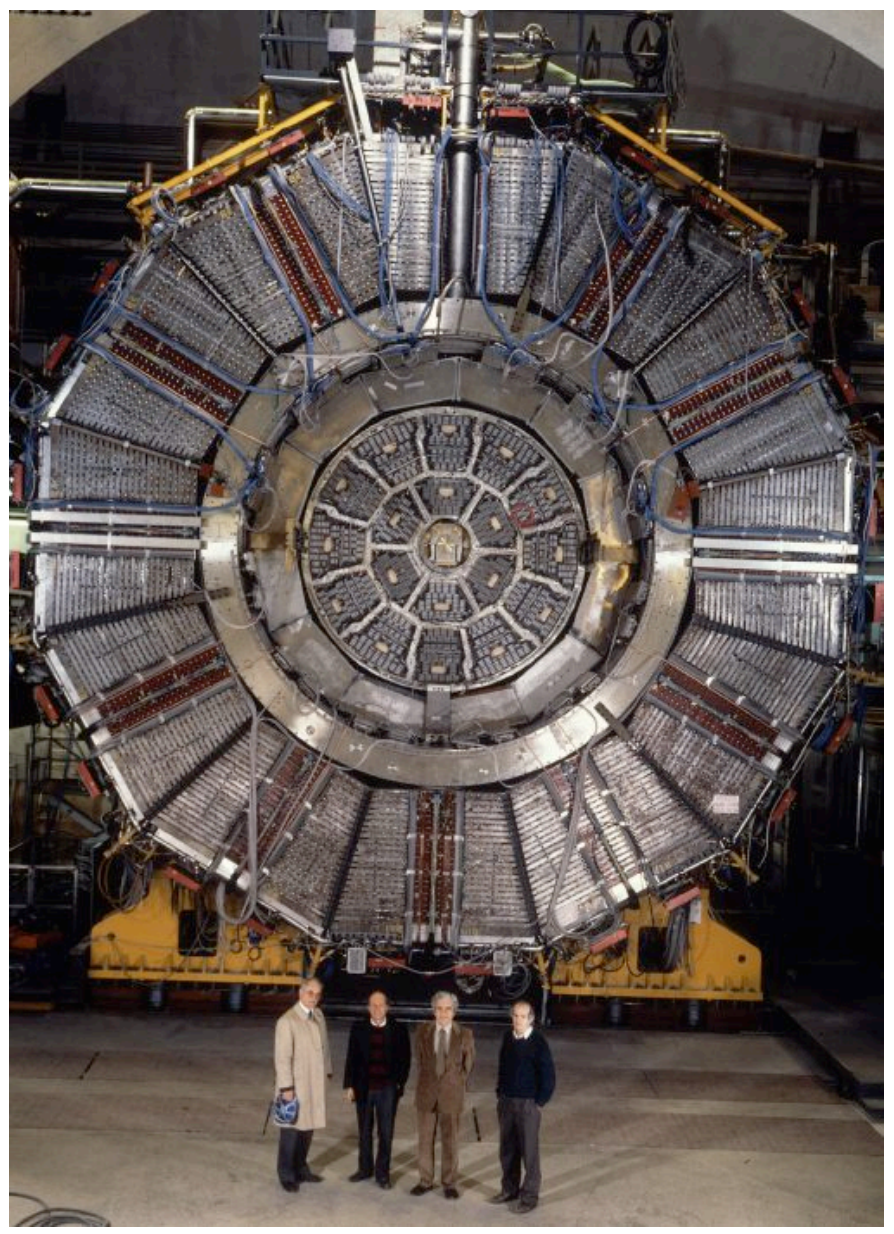

Fig. 25: Front view of the ALEPH detector. 
In OPAL (Fig. 26) [34], the central tracking system consisted of a small radius vertex detector with a cylindrical drift chamber, pressurized to achieve high resolution, a large volume JET chamber providing the main tracking and particle identification, and an outer layer of drift chambers optimised to measure the longitudinal coordinate of tracks (Fig. 27).

\section{OPAL}

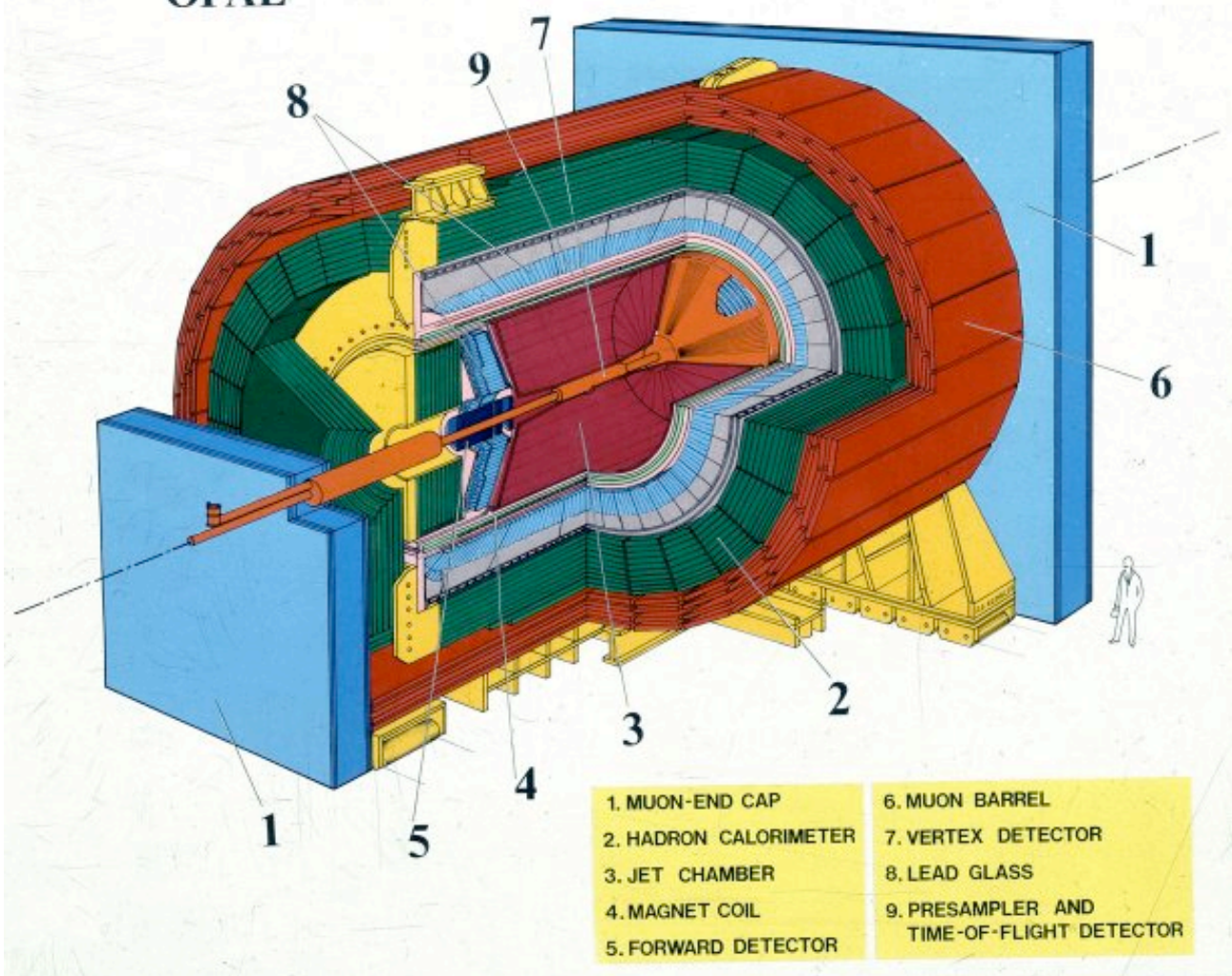

Fig. 26: The OPAL detector.

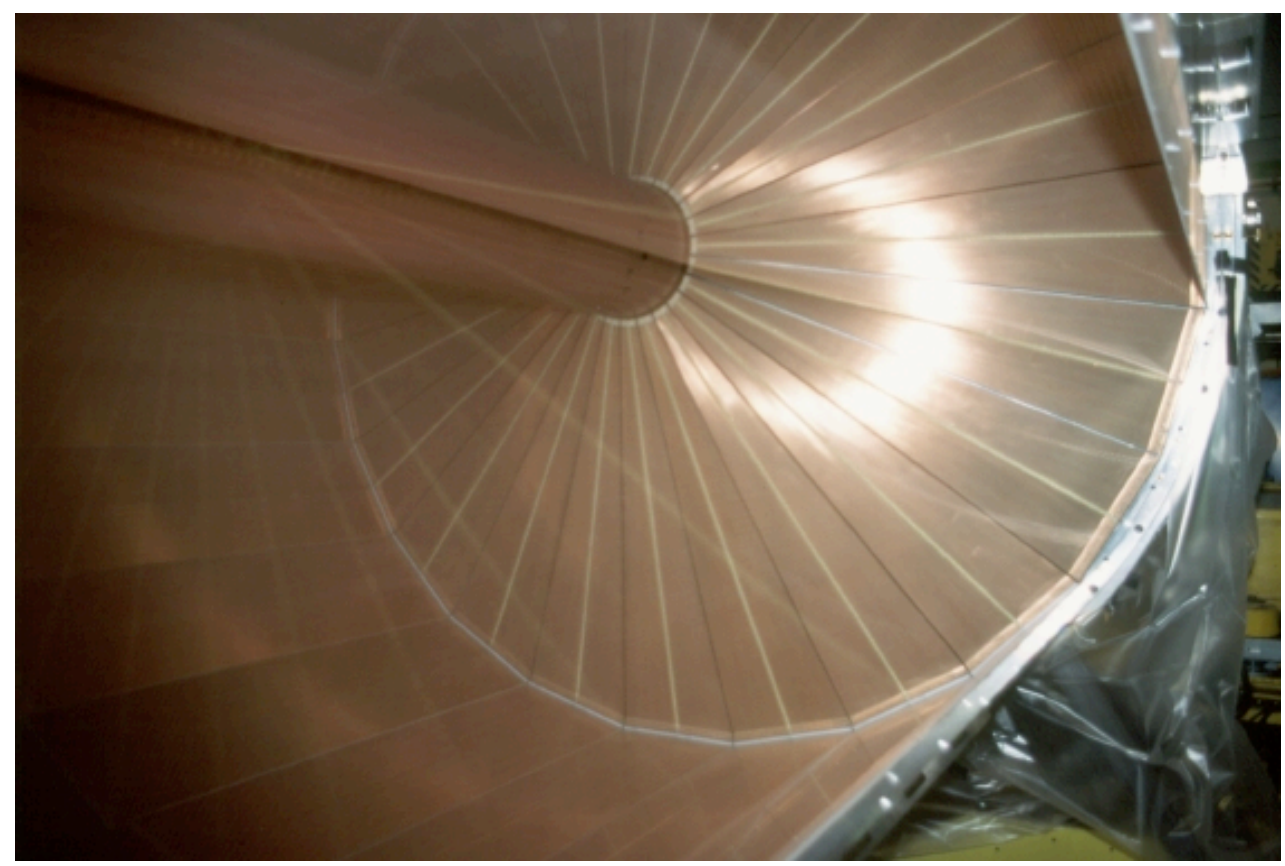

Fig. 27: View inside the OPAL JET chamber. 
The picture in Fig. 28 shows a view into the barrel detector, open during the assembly. Muon detection was done in the barrel region with a set of large area drift chambers, and in the forward directions using layers of limited streamer tubes.

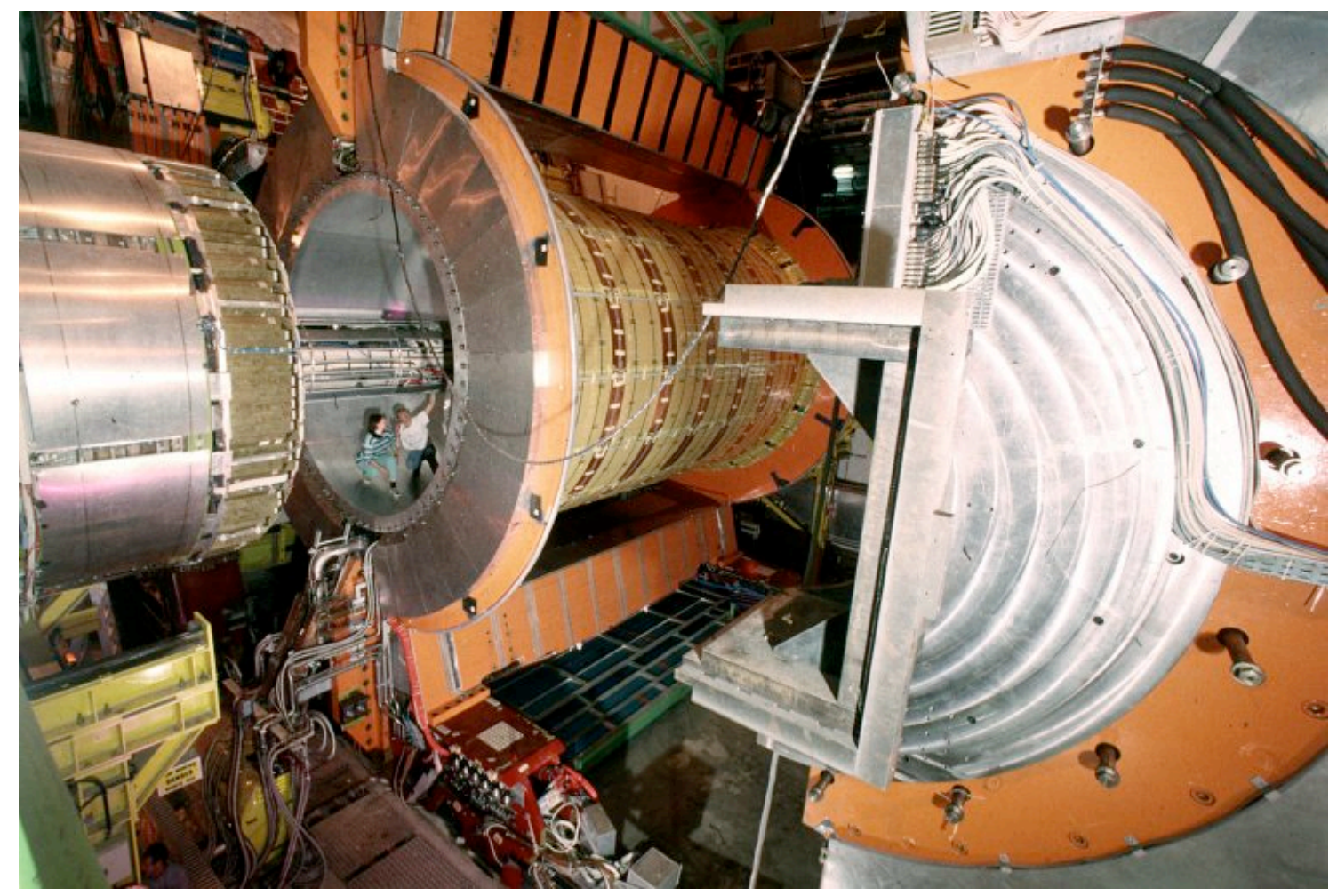

Fig. 28: View into the core of OPAL.

The conception of the fourth experiment, L3, was radically different [35]. To optimize the muon momentum resolution, the experiment used a large volume, moderate field magnet containing the major part of the set-up (Fig. 29). The octagonshaped solenoid had an effective length close to $12 \mathrm{~m}$ and a bore of $11.4 \mathrm{~m}$, and could reach a field of $0.5 \mathrm{~T}$. Close to the beam pipe, a Time Expansion Chamber served as main charged particle tracker, providing the reconstruction of the primary and secondary interaction points, the particle momentum and event multiplicity with a double track resolution better than $500 \mu \mathrm{m}$ [36] (Fig. 30). A BGO crystal calorimeter measured the energy of photons and electrons; hadronic calorimetry was performed with stacks of uranium converters and MWPC planes. Precise muon momentum measurement in the barrel was obtained with several layers of high accuracy drift chambers.

The four experiments took data for more than ten years, providing large amounts of physics information, the most significant being perhaps the confirmation of the existence of only three lepton families, as anticipated by the Standard Model. In December 2000, and despite the finding of a possible signature of the elusive Higgs particle, the operation was stopped and the set-ups disassembled in preparation for the construction of CERN's Large Hadron Collider. 


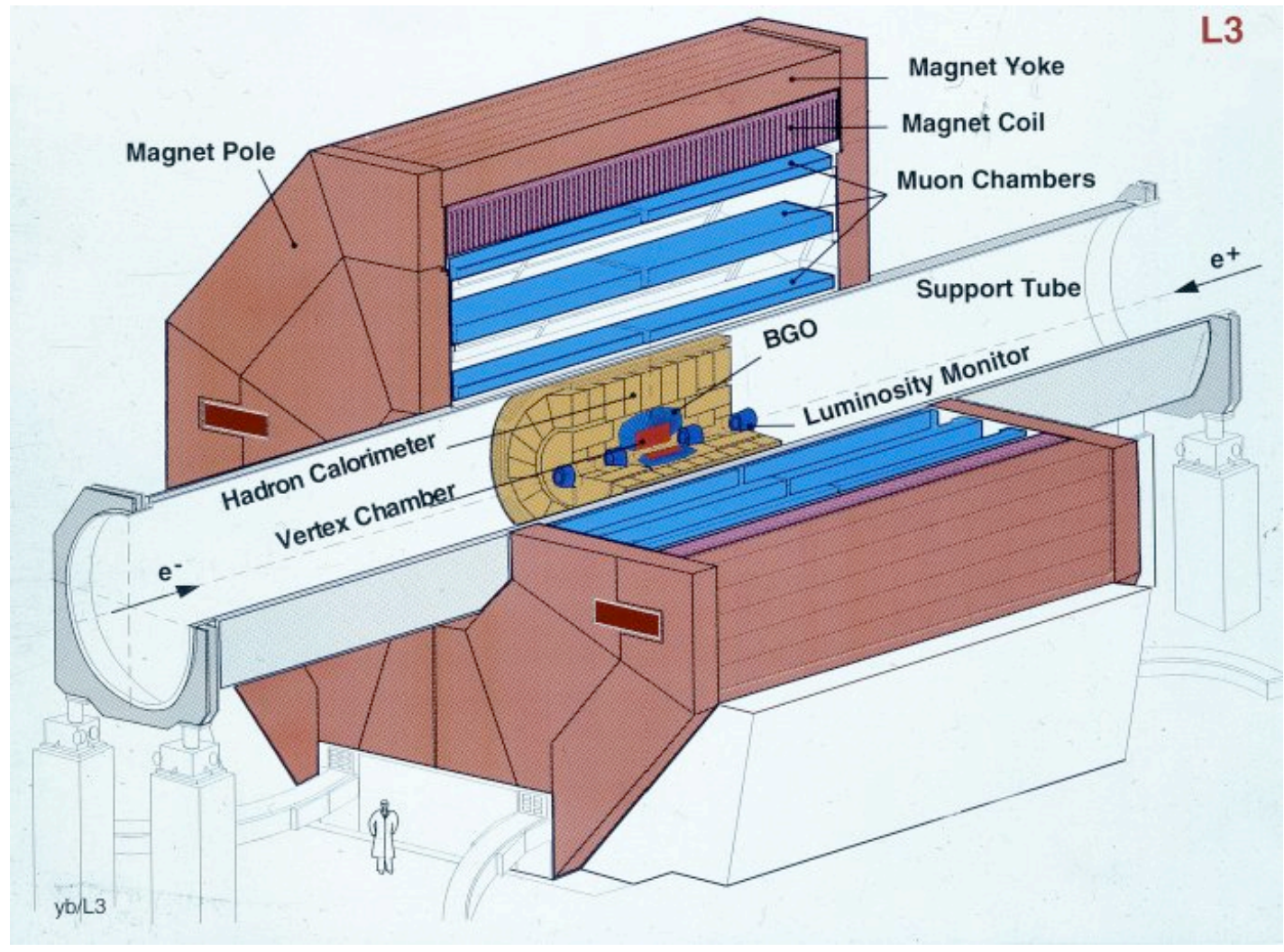

Fig. 29: The L3 experimental set-up.

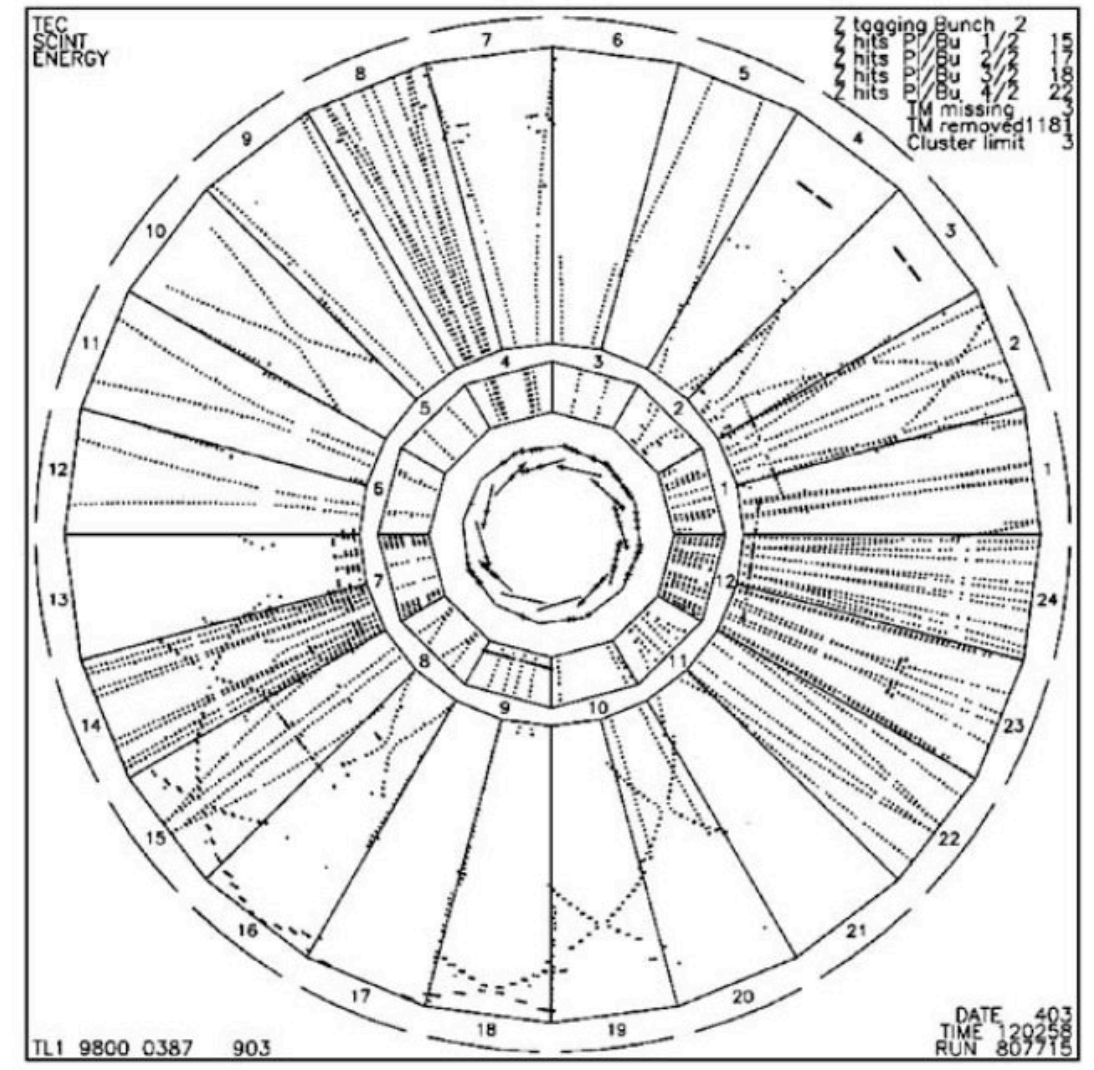

Fig. 30: A multi-track event, recorded with the L3 Time Expansion Chamber. 


\section{PREPARING FOR THE LHC: DETECTOR DEVELOPMENT IN THE NINETIES}

Simultaneously with the exploitation of the large complexes at LEP and fixed target experiments, and in anticipation of the challenging requirements of the forthcoming LHC project, a huge effort was undertaken in the nineties for the development of new technologies. CERN's Research Board approved and supported fifty Detector R\&D projects proposed by international collaborations; a parallel framework, the Lepton Asymmetry Analyzer (LAA) project, based at CERN and independently funded, contributed to this research effort.

With the increase in the available wafer size and the large scale integration of the readout electronics, large arrays of silicon micro-strips became essential components of all major experiments. As an example, Fig. 31 shows a module with two detectors and their readout, developed by the CMS collaboration, manufactured with on 6" wafers. Comparison with Fig. 13 illustrates the substantial progress in the relative areas of sensor and electronics. Matching the high granularity of silicon micro-strip detectors, the readout circuits typically store and multiplex in a single chip the information of 128 adjacent channels; the APV25 chip equipping the silicon strip prototype visible in the picture is a representative example, and is used in several experiments [37]. Recent overviews on the development of silicon detectors can be found in Refs. [38, 39].

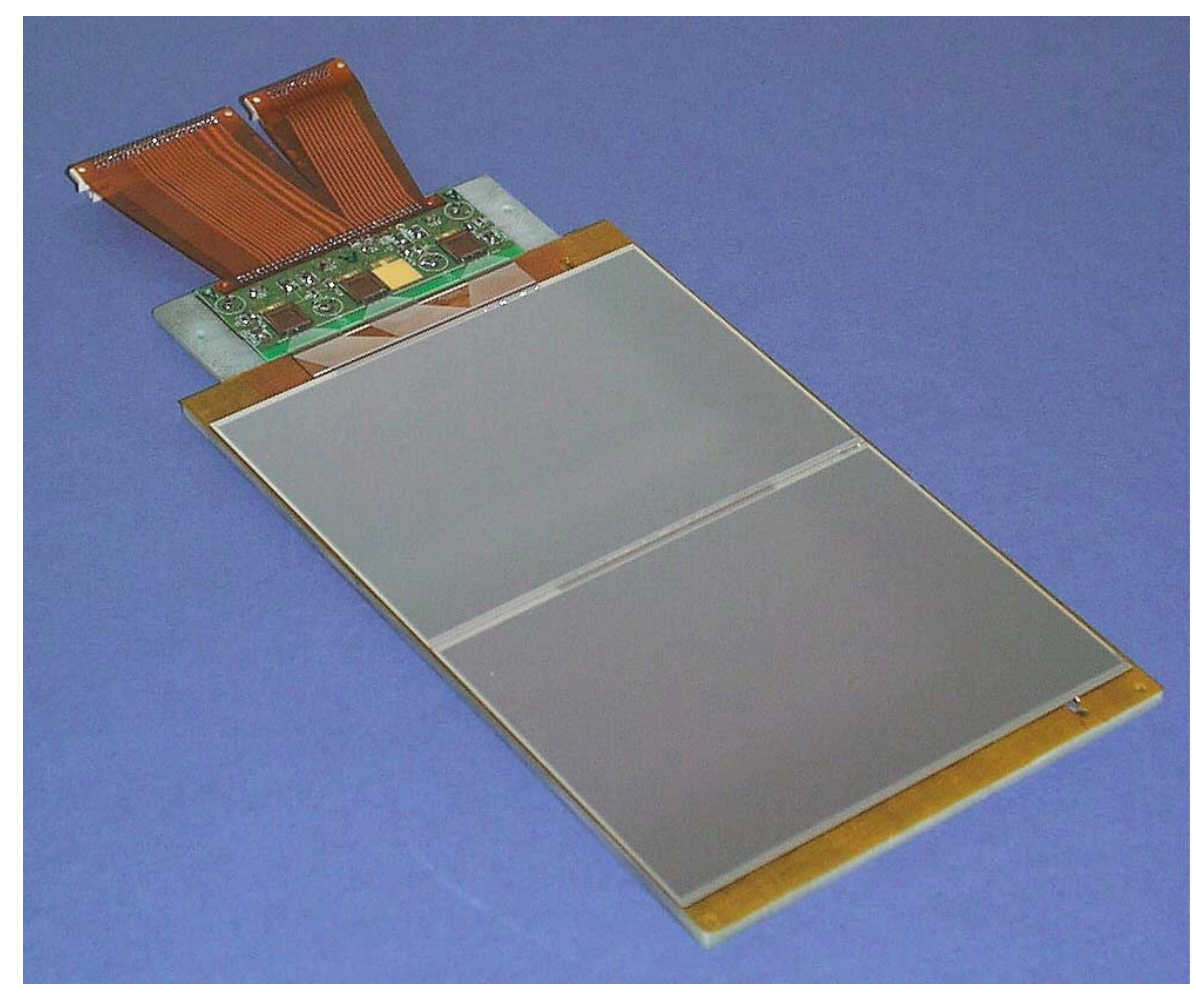

Fig. 31: Twin-wafers silicon micro-strip detectors with readout electronics, prototype for the CMS vertex detector.

With the unprecedented multiplicities and rates expected at future machines, the excessive occupancy of projective readout methods encouraged the development of more powerful devices where small size pixels could be individually read-out, each element acting as an independent detector. Due to very different requirements on the silicon substrate, efforts to integrate sensors and electronics on the same wafer have been only moderately successful, and the common solution is to manufacture 
the two components separately and use an adequate, high-density interconnection technology. One of the methods, developed in the framework of RD19, consists in the formation on the electronics side of soft metal micro-spheres; with a technique called bump-bonding, consisting in the application of a gentle pressure at high temperature, the two plates can be linked together (Fig. 32) [40]. Typical sensors have rectangular or square pixels between 100 and $500 \mu \mathrm{m}$ on the side; Fig. 33 shows a pixel readout chip developed by CERN's micro-electronics group.

Ref. [41].

For a review on pixel detector developments and applications, see for example
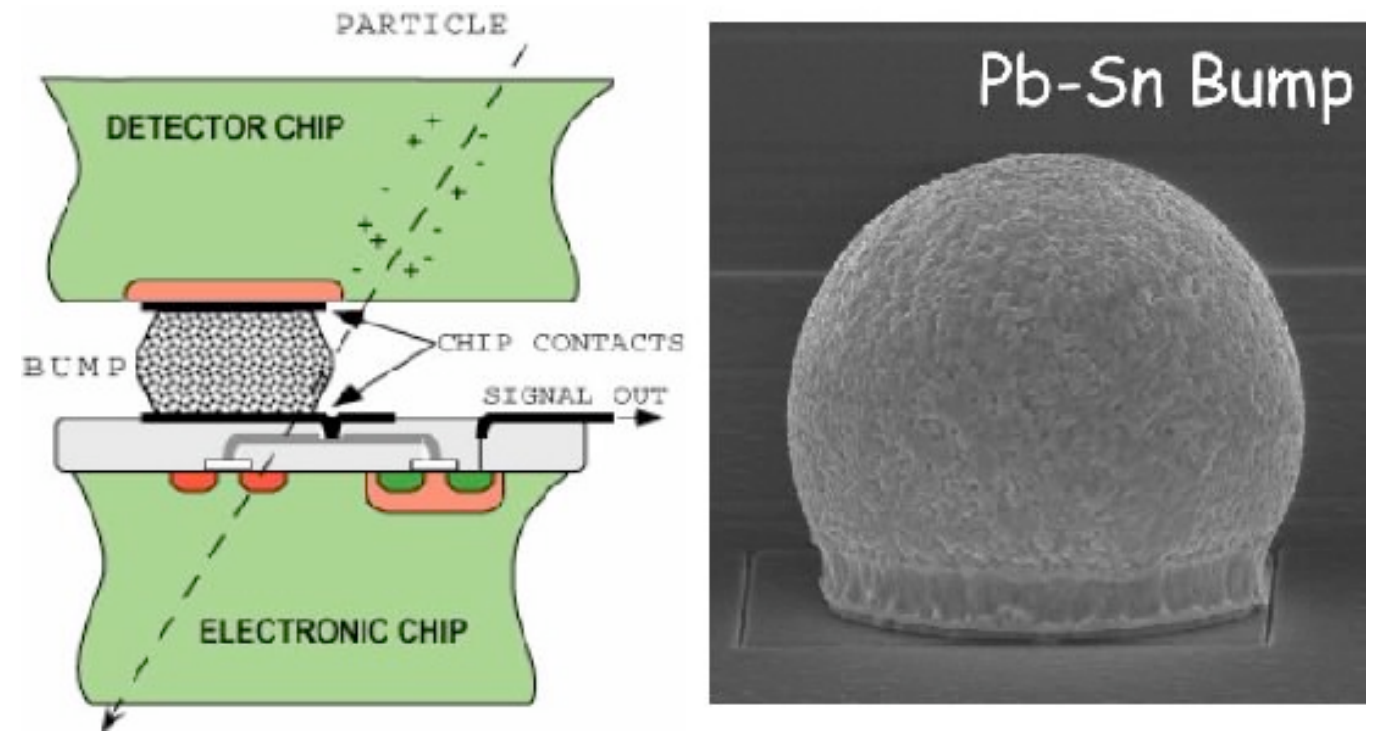

Fig. 32: Schematics of a silicon pixel detector, bump-bonded to the readout electronics, and an electron microscope view of a bump.

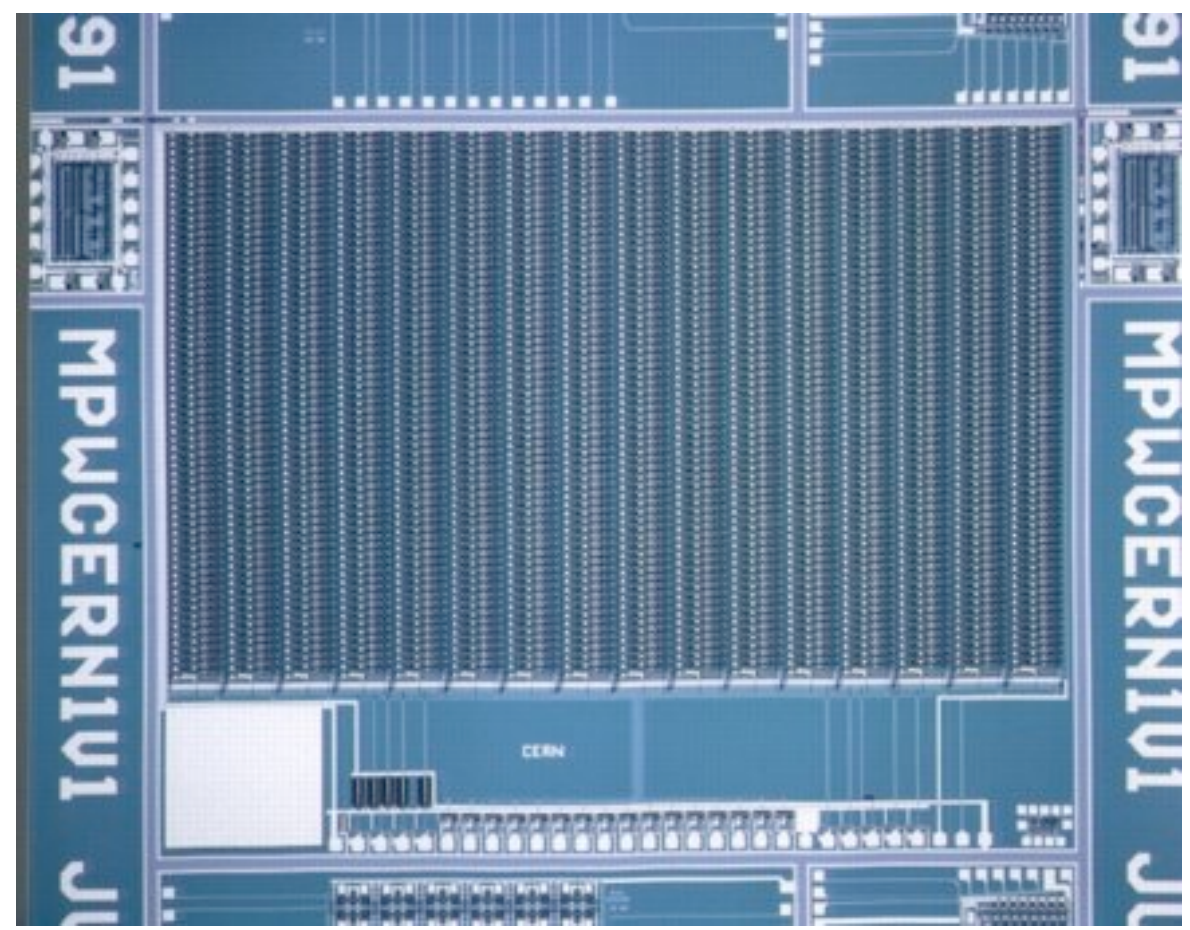

Fig. 33: Silicon pixel readout chip. 
The most serious concern on the use of detectors in the LHC environment is their survivability at extremely high radiation levels. Radiation damage processes in silicon have been extensively studied by the ROSE collaboration (RD48) with exposures to high intensity charged particle, gamma and neutron beams [42]. The results have clearly pinpointed the causes of degradation: physical damage to the crystal structure creating local defects, and global changes in the doping concentration of the material, leading to a gradual type inversion. A major finding was the strong influence of temperature on the rate of damage and operation: cooling the silicon plates results in a considerable lengthening of the detector lifetime. This feature is exploited in the LHC detectors, at the cost of added complexity in the assembly.

Semiconductor materials known to be intrinsically more radiation tolerant than silicon have also been explored; the RD42 collaboration has extensively studied diamond-based detectors [43]. Compound semiconductors, such as cadmium telluride also seem very promising [44] The limited availability of large-scale manufacturing facilities for the new materials has prevented so far a widespread use of the technologies.

As in most cases the readout circuitry is directly mounted on detectors, an intensive survey of electronics capable to survive in the LHC environment has been also conducted. It was found that the deep sub-micron technology, with its small feature size $(0.25 \mu \mathrm{m}$ or less), guarantees acceptable performances at the expected high radiation levels [45].

The development of RICH detectors with a solid photo-sensitive layer deposited on the cathodes of a MWPC permitted much higher time resolutions and rate capabilities than with devices using photosensitive gases [46]. A thin layer of caesium iodide (CsI) appeared the most promising photocathode, thanks to its large quantum efficiency and good stability in time, as demonstrated by the RD26 collaboration. A system of eight large size CsI-RICH chambers equip the fixed target experiment COMPASS at CERN, and started the physics runs in 2002 [47]; Fig. 34 shows a multi-ring event recorded during a high rate run. For a recent survey on the use of RICH systems in particle physics experiments see Ref. [48].
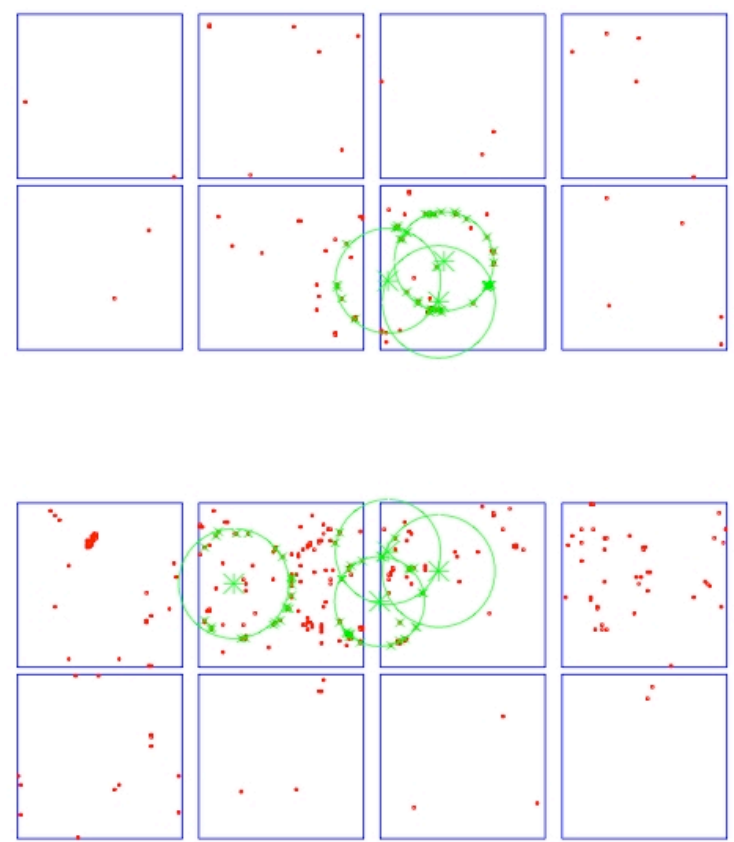

Fig. 34: Multiple ring patterns recorded in the COMPASS RICH. 
The excellent imaging capabilities of the Time Projection Chamber have been demonstrated by the experiment NA49, in the extreme conditions of heavy ion collisions. Their set-up included a sequence of TPC modules, the largest having sensitive volumes of around $100 \mathrm{~m}^{3}$, with a design optimised to handle the very large track multiplicity; Fig. 35 is an impressive view of such an event, recorded by the TPC system.

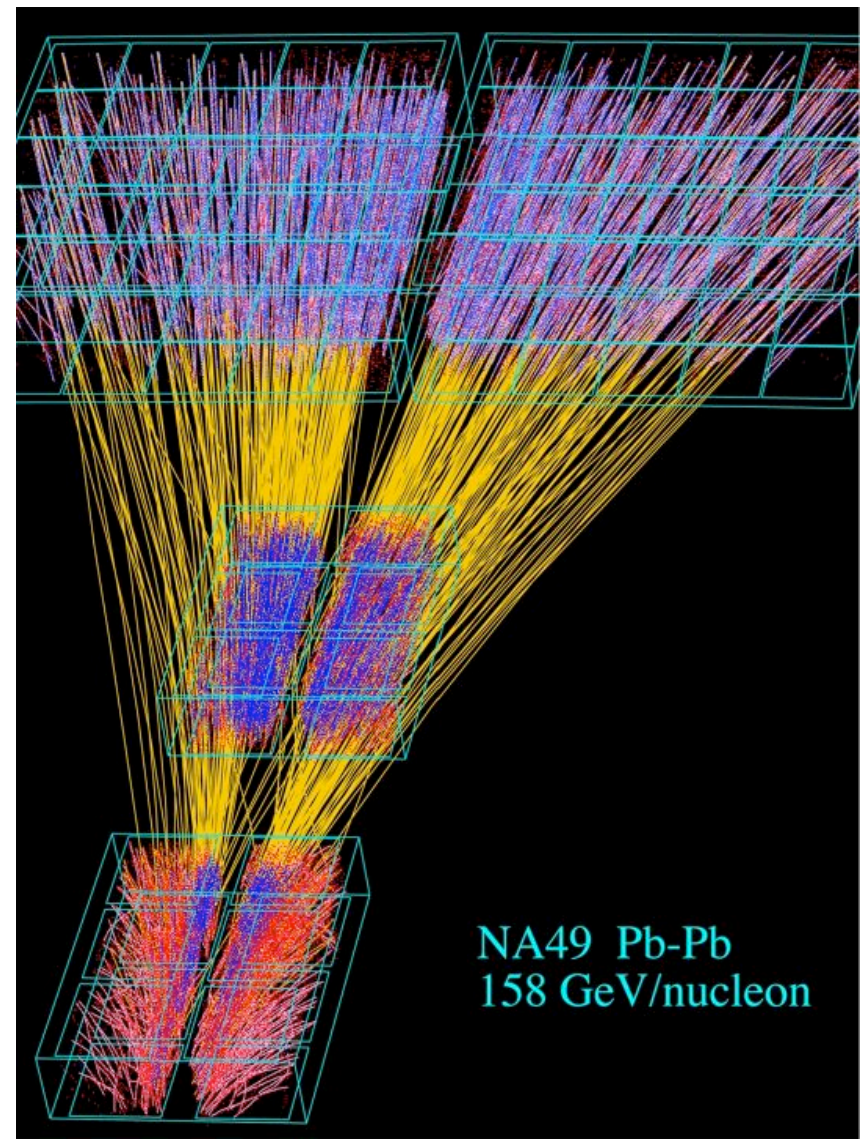

Fig. 35: A high-energy lead-lead collision recorded with the NA49 TPC.

In 1988, Anton Oed introduced a new device, the Micro-Strip Gas Chamber (MSGC), largely outperforming standard wire chambers [49]. Manufactured using precision photolithographic technologies, MSGCs can have a pitch of few hundred microns, largely improving multi-track resolution and rate capability. Unfortunately, they have been found to be easily damaged by discharges, and are used only in a few experiments. Nevertheless, the detailed studies on their properties, and in particular on the radiation-induced processes leading to discharge breakdown led to the development of sturdier devices with similar performances, collectively named Micro-Pattern Gas Detectors (MPGD) [50]. The Gas Electron Multiplier (GEM), developed by the author, consists of a thin metal-coated polymer foil, pierced by a high density of holes (up to a hundred per $\mathrm{mm}^{2}$ ) (Fig. 36) [51]. On application of a potential difference between the two sides, electrons released by ionisation drift into the holes and are amplified by the high local field. Fabricated with simple photolithographic processes, the detector shapes can be tailored to match the experimental requirements. Using a strategy reminiscent of the Multistep Chamber, several GEM foils can be cascaded, permitting to attain large stable gains even in harsh radiation environments [52]. A set of large size Triple-GEM chambers is operating in the COMPASS experiment at CERN, a high rate fixed target spectrometer [47]; Fig 37 shows one of the detectors installed in a test beam line. 


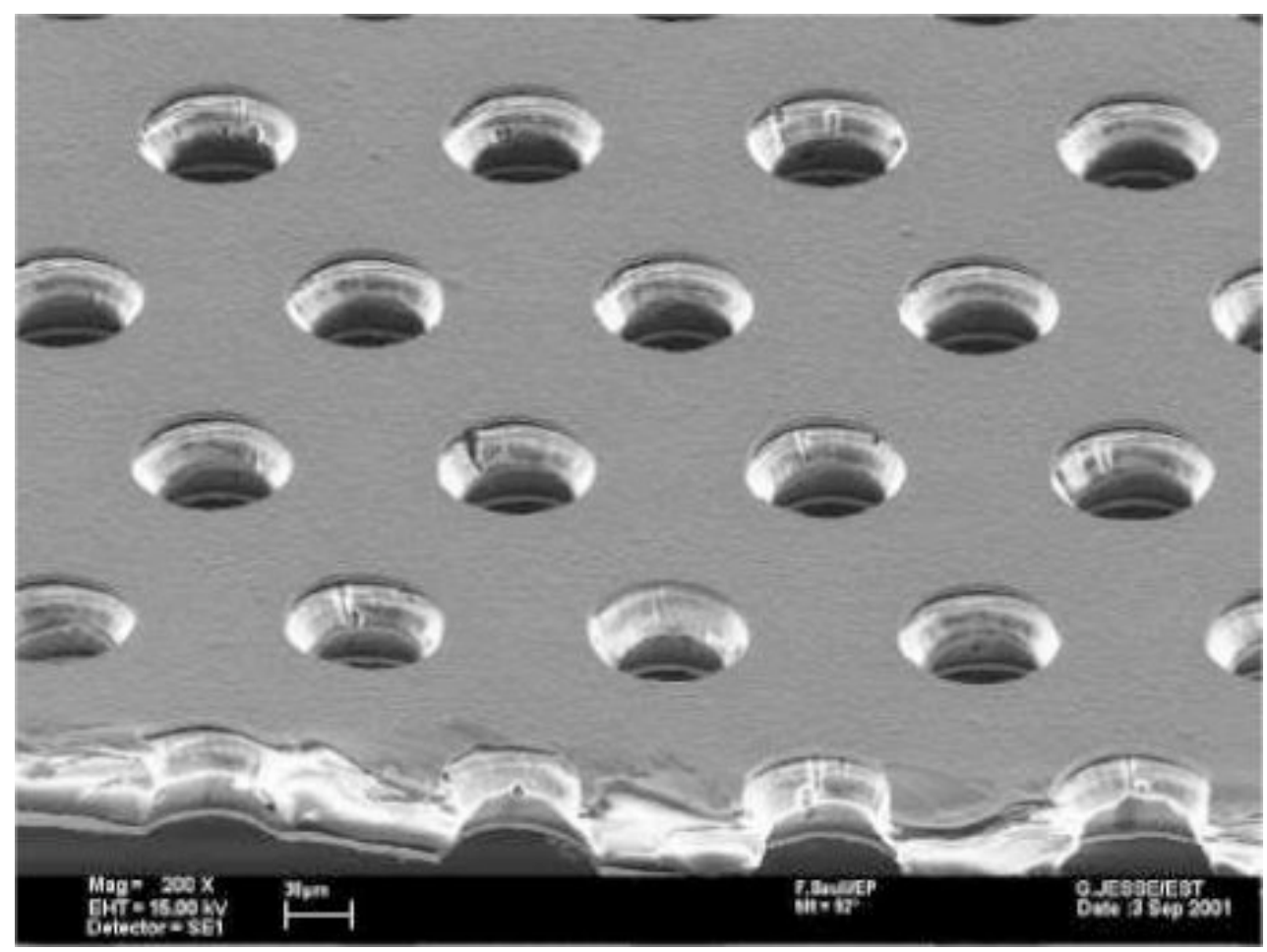

Fig. 36: Electron microscope view of the GEM foil. Each hole acts as an independent gas proportional counter amplifier.

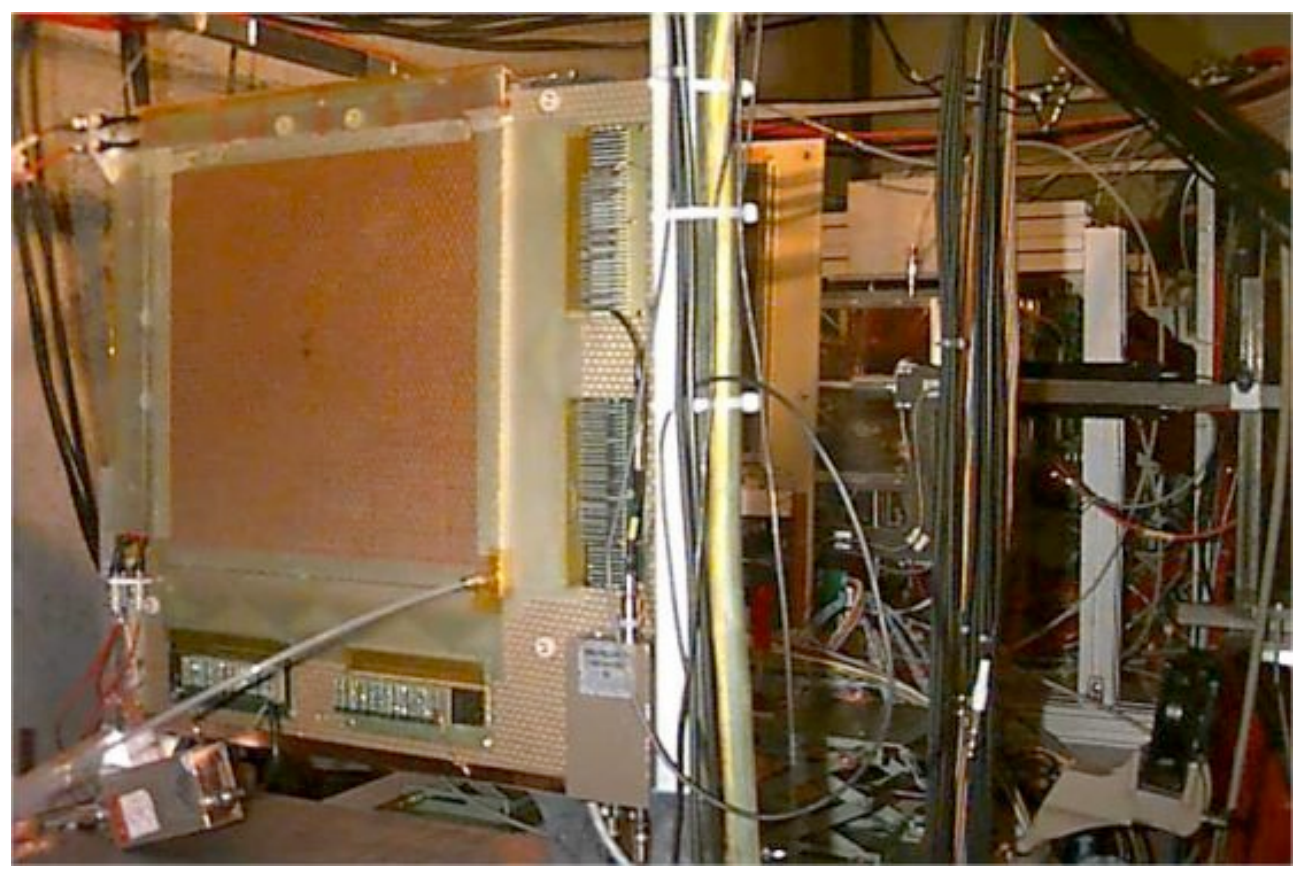

Fig. 37: A large size GEM detector of COMPASS design in the test beam.

Other experiments are developing GEM-based detectors: DESY's Linear Collider, the PHENIX upgrade at BNL, MICE and TOTEM at CERN. Applications in other fields are also under study. As an example, Fig. 38 shows the colour rendering of a micro-mammal absorption X-ray radiography, recorded with a two-dimensional GEM detector [53]. 


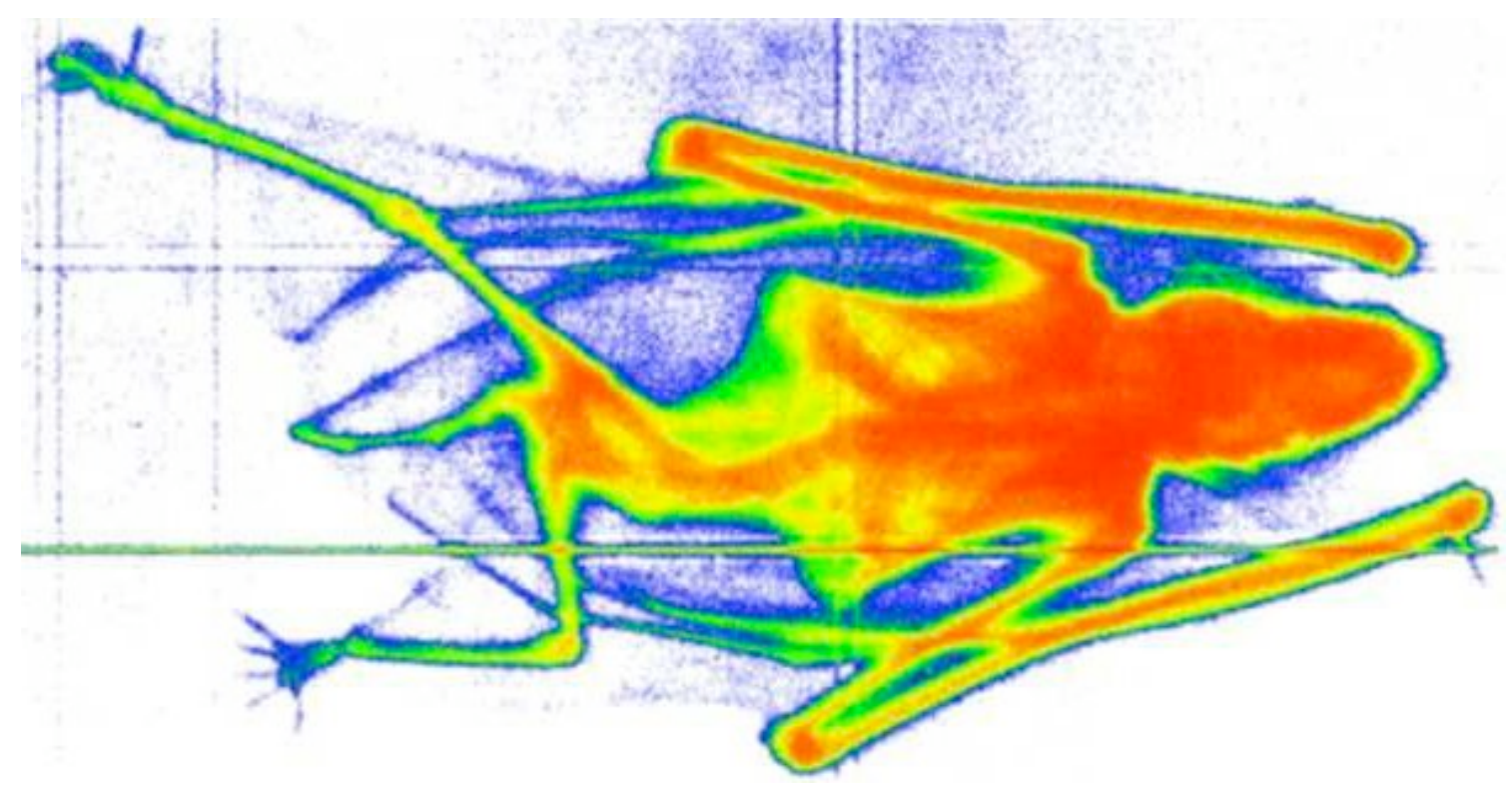

Fig. 38: Digital X-ray radiography of a micro-mammal recorded with a twodimensional GEM detector.

Gaseous detectors degrade when exposed to high radiation levels, a process named aging. This is due to the formation, under the conditions of avalanche multiplication, of polymers of organic gases or of pollutants. Many groups have extensively studied the aging processes, and found that only with very tight requirements on the level of contaminants and of the materials employed for construction the aging rates can be kept at a level compatible with long-term operation. It was also found that carbon-tetra fluoride $\left(\mathrm{CF}_{4}\right)$, used as component of the gas filling, can counteract the polymer deposits with its etching properties; having also a very fast electron drift velocity, gas mixtures containing $\mathrm{CF}_{4}$ appear as ideal candidates for the operation of detectors at high rates. This is the choice made to operate the Transition Radiation Tracker (TRT), a system of thin proportional straw tubes embedded in a light porous material, adopted for the ATLAS experiment [54].

Liquid argon calorimeters record the ionisation released by charged particles in thin liquid-filled parallel gaps interlaced with thick converter plates; widely used in experiments, they provide excellent resolution. To reduce the number of recording channels, modules are usually interconnected in tower-like structures; this becomes progressively difficult with the increasing size of the experiments, and involves long connections degrading the performance. A dedicated research program (RD3) [55], developed an optimised shape for the modules, named accordion, permitting at the same time to decrease the length of interconnections in the towers and to reduce the angular dependence of the response (Fig. 39). For a thorough coverage of various calorimeter technologies see for example Ref. [56].

Organic scintillators are easily damaged by radiation. This has motivated the continuation of the efforts to find alternative inorganic scintillating crystals, intrinsically radiation hard but difficult to produce in large quantity, and has been the subject of research of the Crystal Clear collaboration (RD18). One of the best materials found so far is lead tungstate $\left(\mathrm{PbWO}_{4}\right)$, a transparent crystal having a high density $\left(8.3 \mathrm{~g} \mathrm{~cm}^{-2}\right)$ particularly suited for use in electromagnetic calorimeters, and adopted by several LHC experiments $[57,58]$. The excellent characteristics in revealing hard gamma rays make the new crystals very attractive also for medical diagnosis applications. 


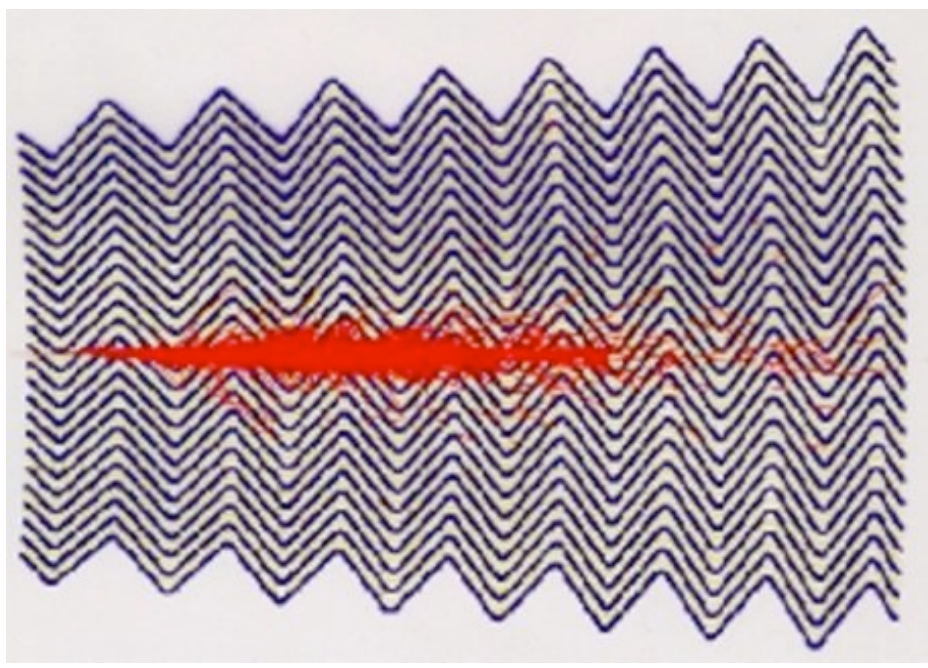

Fig. 39: Schematics of the liquid argon accordion calorimeter, and simulated electro-magnetic shower.

Together with the new scintillating materials, improved devices for the detection of photons have been developed, often in collaboration with industry. Very innovative, the Hybrid Photodiode Detector (HPD) [59], and the Imaging Silicon Pixel Array (ISPA) [60] couple, in the same vacuum envelope, a traditional photocathode with a silicon pixel detector. Photoelectrons ejected by the cathode are focussed and accelerated by a shaped electric field, hitting the detector with energy sufficient to generate detectable signals. HPD prototypes with 5- and 10-inch bi-alkali and solar-blind photocathodes and internal silicon sensors with up to 2048 cells have been manufactured and tested (Fig. 40). With quantum efficiency close to $25 \%$ and single photo-electron sensitivity, the devices are ideal candidates for use in experiments requiring multi-photon imaging capability, both in particle physics and for medical applications.

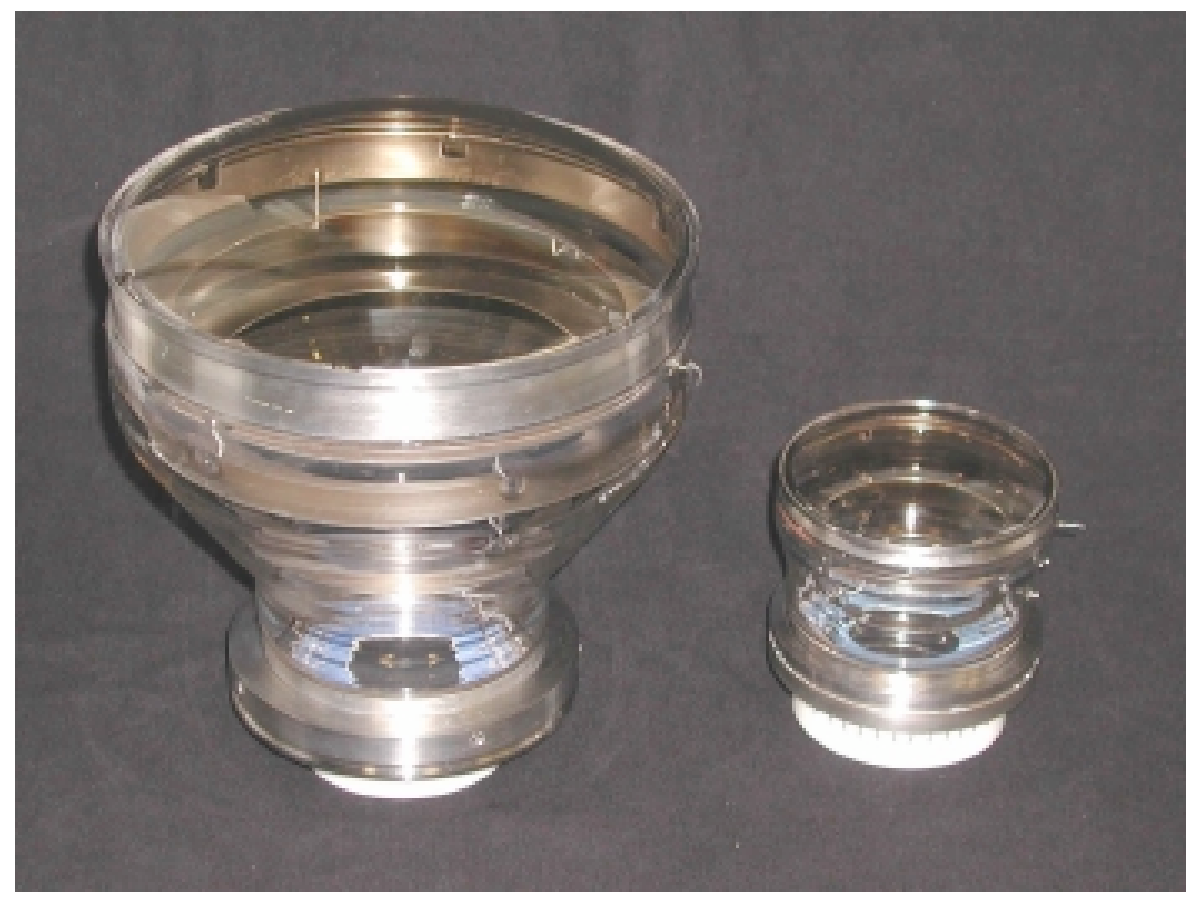

Fig. 40: Prototypes of 10" and 5" Hybrid Photodiodes. 
Although not directly connected to the development of machines and detectors, the World Wide Web, invented at CERN in the early nineties by Tim Berners Lee and Robert Cailliau has undoubtedly become an essential tool for experiment design and control, as well as for data sharing and retrieval (Fig. 41).

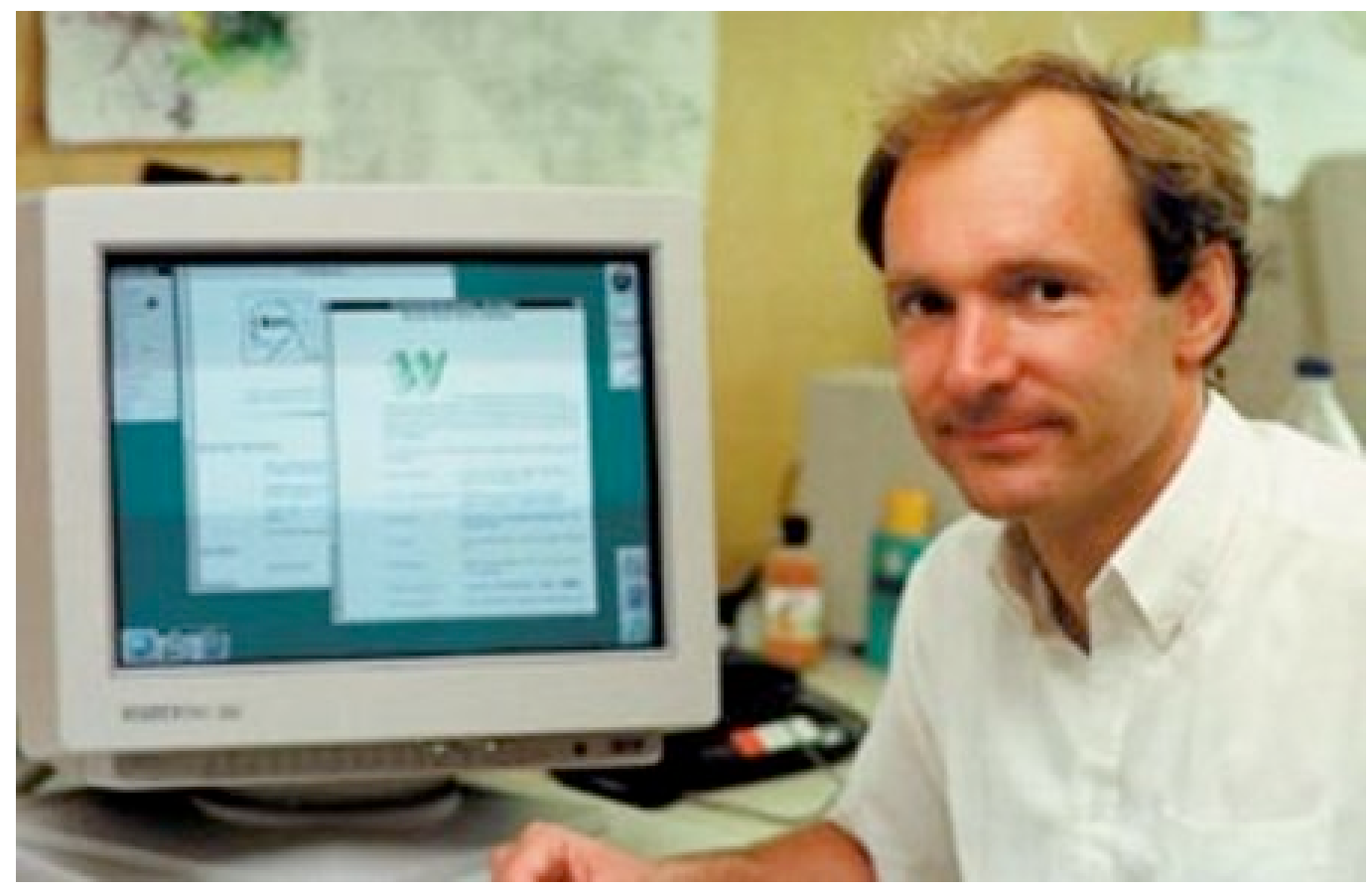

Fig. 41: Tim Berners Lee in 1994 with a display of an early version of a web browser.

\section{LHC: THE CHALLENGE}

In December 1994, the CERN council approved the construction of CERN's new accelerator complex, the Large Hadron Collider (LHC). Hosted in the existing LEP tunnel, it will accumulate and collide two proton beams with $14 \mathrm{TeV}$ in the centre of mass at a design luminosity of $10^{34} \mathrm{~cm}^{-2} \mathrm{~s}^{-1}$. Primarily aimed at finding the Higgs particle, but with a great discovery potential in other fundamental physics domains, the machine will provide bunched proton-proton collisions in four main intersection points. The accelerator will also provide heavy ion collisions (lead on lead) with centre of mass energy up to $1000 \mathrm{TeV}$.

Adding to other sources of background, the radiation in the experimental areas will reach unprecedented levels; this has required a vigorous research to assess the possible effects on the detectors' operation and lifetime.

Four major experiments have been approved and are in various stages of construction [61]. The very difficult access to the set-ups, located in deep underground caverns, and in particular to their inner core, has dictated a strict concern for the reliability of detectors and of the ancillary systems, as well as the development of robust data transmission links and computer control systems. The expected extreme data throughput rates required also the development of advanced technologies for data storage, handling and analysis; CERN's participation in the international GRID project represents a major distributed software development, motivated by the LHC operation.

The LHC experimental set-ups will be only briefly described here, since they are covered in a dedicated article appearing in this volume [62]. Two generalpurpose experimental set-ups, ATLAS (A Toroidal Lhc ApparatuS) and CMS 
(Compact Muon Solenoid), are in construction in their respective surface assembly halls, and will be gradually transferred and re-assembled in the underground caverns. Largely inspired by the LEP experiments, they have an onion-like structure with a succession of detectors both in the barrel and in the forward regions, aimed at determining the event topology, the nature and the energy of emerging particles. In both experiments, the momentum of charged tracks is inferred from the curvature in strong magnetic fields, and particle identification is performed in a succession of electromagnetic and hadronic calorimeters followed by a muon detector.

A dedicated experiment, ALICE (A Large Ion Collider Experiment) has been designed to handle the extremely large multiplicities of tracks produced in heavy ion collisions. The experiment makes use of the large warm magnet recovered from L3, complemented in the forward direction by a dipole to improve the reconstruction of muon pairs. The substantially lower rates permit the use of Silicon Drift Chambers for the inner tracker, and of a Time Projection Chamber as main tracking detector.

Last of the large experiments, LHCb (Large Hadron Collider beauty experiment) is optimised to detect the decay products of B mesons, mostly produced along the beam directions, and is arranged as a single-arm forward spectrometer. The most sophisticated component of the detector is a set of silicon strips entering the vacuum tube of the machine, used to locate the primary and secondary vertices; in their data taking position, the strips descend to a radius only $8 \mathrm{~mm}$ from the beam centre.

At the time of writing (March 2004), the installation of the first dipoles of the machine has begun, and commissioning and starting of physics runs is foreseen for spring 2007.

\section{SUMMARY AND FUTURE PROSPECTS}

Fifty years after its foundation, CERN remains undoubtedly a centre of excellence for research in fundamental particle physics. Experimental set-ups in particle physics have evolved dramatically both in terms of performance and size of the detectors deployed; major components of the experiments are nowadays provided by external institutes, in the framework of world-wide collaborations. This has required the establishment of an intense program of technology transfer, quality control and components integration. New technologies, developed to match specific requirements of the accelerators and of the experiments, have found industrial and commercial applications. Well known examples of such spin-offs are the compact cryogenic magnets widely used in medical imaging, high vacuum technologies, and communication software making the backbone of the Internet. As originally specified in CERN's statute, exploitation of the technologies developed by the laboratory has been traditionally open and free. Recently, an office for Technology Transfer was created with the specific mandate to exploit innovative developments, and to promote the contacts with industrial partners. Example of licensed technologies include methods for treating radioactive waste, active getters used to achieve ultrahigh vacuum, dedicated high-density electronic sensors aimed at medical applications, improved position-sensitive detectors, circuit interconnection technologies.

The experimenter's team size has followed the increasing dimension of the set-ups, reaching unprecedented levels. Requiring complex managerial structures, this evolution is prone to generate relational problems difficult to resolve. The long duration of the projects, measured in decades, together with the extreme specialization of the groups, represent a challenge for preserving the continuity of competence and the long-term maintenance of the set-ups. 
In the last decade, the extreme challenge presented by the design and construction of the new accelerator complex and of the large detectors dragged most of the available resources and intellectual energy of CERN and contributing institutions. This has undoubtedly gone at the expense of other directions of research, often reduced for lack of funding and manpower. Possible negative longterm consequences of these restrictions, particularly in view of new future challenges, have been recognized by the scientific management, with the creation of several programs aimed at preserving CERN's level of excellence in the future. This commitment can be exemplified with a quote from the address to the staff on January 13, 2004 by Robert Aymar, taking charge as CERN's Director General:

"The CERN LHC project is the worldwide priority in high-energy physics: the support of CERN for this objective will not fade out. The limited reduction in the scientific activities at CERN during the LHC construction is the price to pay for the future possession of this powerful tool.

Nevertheless, big challenges are ahead of us, which will require hard work and complete dedication. You can be proud of the results achieved so far, both machinewise, in experiments, and in GRID development I am fully confident in the motivation and competences of each of you. I am convinced that the challenges ahead of us will be overcome:

- The LHC can be ready in time (within cost) and will be another success in the large series of CERN prestigious achievements: scientific discoveries will not be far ahead,

- The Members States' confidence in CERN can be fully restored, around an agreed European scientific strategy, supported by appropriate resources, thus providing CERN with a sure future."

\section{REFERENCES}

[1] L. Van Hove and M. Jacobs, Highlights of 25 years of physics at CERN, Physics Reports 62 (1980) 1.

[2] J. Krige, ed., History of CERN Vol. III (Elsevier, Amsterdam 1996).

[3] R. Beuselinck et al., Performance of the External Muon Identifier for the Big European Bubble Chamber, Nucl. Instr. and Meth. 154 (1978) 445.

[4] P. Rice-Evans, Spark, Streamer, Proportional and Drift Chambers (Richelieu, London, 1974).

[5] F. Rohrbach, Streamer Chambers at CERN During the past Decade and Visual Techniques of the Future, CERN / EF 88-17 (1988).

[6] G. Charpak et al., The use of multiwire proportional counters to select and localize charged particles, Nucl. Instr. and Meth. 62 (1968) 262.

[7] G. Charpak and F. Sauli, High-resolution electronic particle detectors, Ann. Rev. Nucl. Part. Sci. 34 (1984) 285.

[8] P. Schilly et al., Construction and performance of large Multiwire Proportional Chambers, Nucl. Instr. and Meth. 91 (1971) 221.

[9] R. Bouclier et al., Proportional Chambers for a 50 000-wire detector, Nucl. Instr. and Meth. 115 (1974) 235.

[10] A.H. Walenta et al., The Multiwire Drift Chamber: a new type of Proportional Wire Chamber, Nucl. Instr. and Meth. 92 (1971) 373.

[11] G. Marel et al., Large Planar Drift Chambers, Nucl. Instr. and Meth. 141 (1977) 43.

[12] A.N. Diddens et al., A detector for neutral-current interactions of high energy neutrinos, Nucl. Instr. and Meth. 178 (1980) 27.

[13] D. Nygren, Future prospects of the Time Projection Chamber, Phys. Scripta 23 (1981) 584. 
[14] E. Radermacher, The experimental discovery of the intermediate vector bosons $\mathrm{W}^{+}$, $\mathrm{W}^{-}$and $\mathrm{Z}_{0}$ at the CERN proton-antiproton collider, Prog. Part. Nucl. Phys. 14 (1985) 231.

[15] P. Baussgessner et al., The UA1 Central Detector at High Luminosity (ACOL), Nucl. Instr. and Meth. in Phys. Res. A257 (1987) 552.

[16] E.H.M. Heijne et al., A silicon surface barrier microstrip detector for high energy physics, Nucl. Instr. and Meth. 178 (1980) 331.

[17] E. Beauville et al., AMPLEX, a low-noise, low-power analog CMOS signal processor for multi-element silicon particle detectors, Nucl. Instr. and Meth. in Phys. Res. A288 (1990) 157.

[18] R. Klanner, Silicon detectors, Nucl. Instr. and Meth. in Phys. Res. A235 (1985) 209.

[19] A.H. Walenta, The Time Expansion Chamber and Single ionization Cluster Mesurement, IEEE Trans. Nucl. Sci. NS-26 (1979) 73.

[20] J. Seguinot and T. Ypsilantis, Photo-ionization and Cherenkov ring imaging, Nucl. Instr. and Meth. 142 (1977) 377.

[21] J. Seguinot and T. Ypsilantis, A historical survey of ring imaging Cherenkov counters, Nucl. Instr. and Meth. in Phys. Res. A343 (1994) 1.

[22] G. Charpak and F. Sauli, The multistep avalanche chamber: a new high rate, high accuracy gaseous detector, Phys. Letters 78 B (1978) 523.

[23] R. McCarty et al., Identification of large transverse momentum hadrons using a RingImaging Cherenkov Counter, Nucl. Instr. and Meth. in Phys. Res. A248 (1986) 69.

[24] D. Anderson, Phys. Lett. 118 B (1982) 230.

[25] R. Baur et al., The CERES RICH detector, Nucl. Instr. and Meth. in Phys. Res. A343 (1994) 87.

[26] T. Ekelof et al., The Cherenkov Ring Imaging detector: recent progress and new developments, Phys. Scripta 23 (1981) 718.

[27] H.W. Siebert et al., The Omega RICH, Nucl. Instr. and Meth. in Phys. Res. A343 (1994) 60.

[28] A.P. Jeavons et al., The high-density multiwire drift chamber, Nucl. Instr. and Meth. 124 (1975) 491.

[29] H.G. Fischer, The DELPHI High Density Projection Chamber, Nucl. Instr. and Meth. in Phys. Res. A265 (1988) 218.

[30] C. Fabjan, Calorimetry in High Energy Physics, in Experimental Techniques in High Energy Physics, T. Ferbel, Editor, (Addison-Wesley, Menlo Park, 1985) p. 257.

[31] E. Lorenz, Status of BGO developemnt and perspectives of BGO calorimetrers in High Energy Physics, Nucl. Instr. and Meth. in Phys. Res. 225 (1984) 500.

[32] DELPHI Collaboration, The DELPHI detector at LEP, Nucl. Instr. and Meth. in Phys. Res. A303 (1991) 233.

[33] D. Decamp et al., ALEPH: A detector for electron-positron annihilations at LEP, Nucl. Instr. and Meth. in Phys. Res. A294 (1990) 121.

[34] OPAL Collaboration, The OPAL detector ar LEP, Nucl. Instr. and Meth. in Phys. Res. A305 (1991) 275.

[35] B. Adeva et al., The construction of the L3 experiment, Nucl. Instr. and Meth. in Phys. Res. A289 (1990) 35.

[36] H. Anderhub et al., Experience with the L3 vertex drift chamber at LEP, Nucl. Instr. and Meth. in Phys. Res. A515 (2003) 31.

[37] M.J. French et al., Design and results from the APV25, a deep sub-micron CMOS front-end chip for the CMS tracker, Nucl. Instr. and Meth. A 466 (2001) 359.

[38] P. Weilhammer, Overview: silicon vertex detectors and trackers, Nucl. Instr. and Meth. in Phys. Res. A453 (2000) 60. 
[39] H. Dyjkstra, Overview of silicon detectors, Nucl. Instr. and Meth. in Phys. Res. A478 (2002) 37.

[40] P. Delpierre et al., Development of silicon micropattern (pixel) detectors, Nucl. Instr. and Meth. in Phys. Res. A315 (1992) 133.

[41] N. Wermes, Pixel detectors for particle physics and imaging applications, Nucl. Instr. and Meth. in Phys. Res. A512 (2003) 277.

[42] G. Lindström et al., Developments for radiation hard silicon detectors by defect engineering-results by the CERN RD48 (ROSE) Collaboration, Nucl. Instr. and Meth. in Phys. Res. A465 (2001) 60.

[43] W. Adam et al., Status of the R\&D acrivity on diamond particle detectors, Nucl. Instr. and Meth. in Phys. Res. A511 (2003) 124.

[44] P. Sellin, Recent advances in compound semiconductor radiation detectors, Nucl. Instr. and Meth. in Phys. Res. A513 (2003) 332.

[45] W. Snoeys et al., Pixel readout chip in deep submicron CMOS for ALICE and LHCb tolerant to $10 \mathrm{Mrad}$ and beyond, Nucl. Instr. and Meth. in Phys. Res. A466 (2001) 366.

[46] J. Séguinot et al., Reflective UV photocathodes with gas-phase electron extraction: solid, liquid, and adsorbed thin films, Nucl. Instr. and Meth. in Phys. Res. A297 (1990) 133.

[47] G.K. Mallot, The COMPASS spectrometer at CERN, Nucl. Instr. and Meth. in Phys. Res. A518 (2004) 121.

[48] F. Piuz, Ring Imaging Cherenkov systems based on gaseous photo-detectors: trends and limits around particle physics accelerators, Nucl. Instr. and Meth. in Phys. Res. A502 (2003) 76.

[49] A. Oed, Position-Sensitive Detector With Micro-strip Anode for Electron Multiplication with Gases, Nucl. Instr. and Meth. A263 (1988) 351.

[50] F. Sauli and A. Sharma, Micro-Pattern Gaseous Detectors, Ann. Rev. Nucl. Part. Sci. 49 (1999) 341.

[51] F. Sauli, GEM: A new concept for electron amplification in gas detectors, Nucl. Instr. and Meth. A386 (1997) 531.

[52] C. Altunbas et al., Construction, test and commissioning of the Triple-GEM tracking detector for COMPASS, Nucl. Instr. and Meth. A490 (2002) 177.

[53] F. Sauli, Development and applications of gas electron multiplier detectors, Nucl. Instrum. Methods A505 (2003) 195.

[54] T. Akesson et al., Status of design and construction of the Transition Radiation Tracker (TRT) for the ATLAS experiment at the LHC, Nucl. Instr. and Meth. in Phys. Res. In press (2004).

[55] B. Aubert et al., Performance of a liquid argon electromagnetic calorimeter with an "accordion" geometry, Nucl. Instr. and Meth. in Phys. Res. A309 (1991) 438.

[56] C.W. Fabjan and F. Gianotti, Calorimetry for Particle Physics, Rev. Modern Phys. 75 (2003) 1243.

[57] A.A. Annenkov et al., Lead tungstate scintillation material, Nucl. Instr. and Meth. in Phys. Res. A490 (2002) 30.

[58] E. Auffray et al., Crystal conditioning for high-energy physics detectors, Nucl. Instr. and Meth. in Phys. Res. A 486 (2002) 22.

[59] A. Braem et al., Development of a 10-inch HPD with integrated readout electronics, Nucl. Instr. and Meth. in Phys. Res. A504 (2003) 19.

[60] T. Gys et al., A new position-sensitive photon detector based on an imaging silicon pixel array (ISPA-tube), Nucl. Instr. and Meth. in Phys. Res. A355 (1995) 386.

[61] M. Price, The LHC project, Nucl. Instr. and Meth. in Phys. Res. A478 (2002) 46.

[62] T. Virdee, Detectors for LHC, Physics Reports This issue (2004). 